\title{
X-ray absorption spectroscopy through damped coupled cluster response theory
}

\author{
Thomas Fransson
}

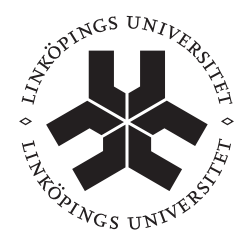

\section{Linköpings universitet INSTITUTE OF TECHNOLOGY}

\author{
LIU-TEK-LIC-2013:59
}

Department of Physics, Chemistry, and Biology (IFM) Linköping University, SE-581 83 Linköping, Sweden 
ISBN 978-91-7519-484-4

ISSN 0280-7971

Printed by LiU-Tryck 2013 
For a fundamental understanding of the interaction of electromagnetic radiation and molecular materials, experimental measurements are to be combined with theoretical models. With this combination, materials can be characterized in terms of composition, structure, time-resolved chemical reactions, and other properties. This licentiate thesis deals with the development and evaluation of a theoretical method by which X-ray absorption spectra can be interpreted and predicted.

In X-ray absorption spectroscopy the photon energy is tuned such that core electrons are targeted and excited to bound states. Such core excitations exhibit strong relaxation effects, making theoretical considerations of the processes especially challenging. In order to meet these challenges, a damped formalism of the coupled cluster (CC) linear response function has been developed, and the performance of this approach evaluated. Amongst the quantum chemical methods available, CC stands out as perhaps the most accurate, with a systematic manner by which the correct physical description can be approached. Coupled with response theory, we thus have a reliable theoretical method in which relaxation effects are addressed by means of an accurate treatment of electron correlation.

By use of the hierarchy of CC approximations (CCS, CC2, CCSD, CCSDR(3)), it has been shown that the relaxation effects are accounted for by the inclusion of double and triple excitations in the CC excitation manifold. The performance of the methods for $K$-edge NEXAFS spectra for water, neon, carbon monoxide, ammonia, acetone, and a number of fluorine-substituted ethenes has been investigated, and we observe relaxation effects amounting to $7-21 \mathrm{eV}$. The discrepancy in absolute energy for the most accurate calculations as compared to experiments are reported as $0.4-1.5 \mathrm{eV}$, and the means by which this can be decreased further are discussed. For relative energies, it has been demonstrated that CCSD yields excellent spectral features, while $\mathrm{CC} 2$ yields good agreement to experiments only for the most intense features. Comparisons have also been made to the more computationally viable method of density functional theory, for which spectral features are in excellent agreement with experiment. 


\section{Acknowledgements}

First of all, I would like to thank my many friends and colleagues at IFM for making this first half of the journey towards a Ph.D. fly by with (relative) ease. The fika sessions discussing something completely random, hours spent trying to figure out which restaurant offers the most appealing meal of the day and so on are always much appreciated. For that I would like to extend special thanks to Jonas Sjöqvist, whose supply of odd topics for discussion, candy and opinions about restaurants seems to have no limit. I would also like to thank Joanna Kauczor for her ability to lighten up the occationally dull corridor and for, along with Mathieu Linares, helping me find my role model.

Having spent the last year and a half visiting more airports than the rest of my life taken all together, I would also like to thank the people who made these trips worthwhile: My colleagues in the beautiful German city of Heidelberg for making me feel welcome during my weeks there and showing me around, especially Dirk Rehn, and Andreas Dreuw for inviting me. The teachers and fellow participants of the summer school in Geneva 2013 and the school here in Linköping at the fall of 2012. The teachers and participants of the summerschool (note the spelling) in Sicily 2013, and then especially my comrades from IFM, Tobias Fahleson and Riccardo Volpi, who joined me in this trip so that we could delve deeply into the fundamentals of quantum chemistry. As well as the noble art of Italian hand gesturing. For the winter school in a surprisingly snowy Stockholm 2012, I would again like to thank the teachers and my fellow participants, in the latter group especially my collegues from home, Paulo V C Medeiros and Cecilia Goyenola, who made the chilly mornings waiting for the bus seem much warmer.

As doing research, going for fika and attending courses are only three of the four fundamental components of the life of a Ph.D. student, I also take this opportunity to thank my students in the electromagnetism course, for making teaching both a challenge and a joy. 
Outside of academia, I would like to thank my friends and family for supporting me and making life so much more enjoyable. I know that some of you are every so slightly confused as to what I actually do all days, but I hope that we will be able to sort this out one day.

Last, but by no means least, I would like to thank my supervisor, Patrick Norman, for his great patience, knowledge and enthusiasm. Thank you.

Thomas Fransson

Linköping, November 2013 
1 Introdution 1

2 Electronic structure theory $\quad \mathbf{5}$

2.1 Fundamentals of electronic structure theory . . . . . . . . . 6

2.1.1 Ab initio electron wave function methods . . . . . . . . . 6

2.1.2 Relativistic effects . . . . . . . . . . . . . . 7

2.1.3 Approaching the correct wave function . . . . . . . . . 8

2.2 Coupled cluster theory . . . . . . . . . . . . . . . . . . . . . 9

2.2.1 The exponential ansatz . . . . . . . . . . . 10

2.2.2 Approximate coupled cluster methods . . . . . . . . . . 12

2.2.3 Illustrative calculations on neon . . . . . . . . . . 13

3 Molecular response theory $\quad 15$

3.1 Exact state response theory . . . . . . . . . . . . . . 15

3.1.1 General response theory . . . . . . . . . . . . . 16

3.1.2 Damped linear response theory . . . . . . . . . . . . 17

3.1.3 Quasi-energy formalism . . . . . . . . . . . . . 21

3.2 Damped coupled cluster linear response theory . . . . . . . . . 23

3.2.1 Damped coupled cluster linear response function . . . . . . 23

3.2.2 The asymmetric Lanczos-chain method . . . . . . . . . . . 27

3.2.3 Direct solver in a reduced subspace . . . . . . . . . . . 30

3.2.4 Comparison of the Lanczos and reduced subspace approaches 32

4 Results and discussion 35

4.1 X-ray absorption spectroscopy . . . . . . . . . . . . . . 35

4.1.1 Performance of the coupled cluster hierarchy . . . . . . . 36

4.1.2 Treating larger molecules . . . . . . . . . . . . . 42

4.2 Other applications using damped coupled cluster response theory . $\quad 45$

4.2.1 Absorption and dispersion of ultraviolet/visible radiation . 46 
4.2.2 Dipole-dipole dispersion coefficients . . . . . . . . . . 47

5 Conclusions $\quad 49$

$\begin{array}{ll}\text { Bibliography } & 51\end{array}$

List of included Publications $\quad 55$

$\begin{array}{lr}\text { Paper I } & 57\end{array}$

$\begin{array}{lr}\text { Paper II } & 67\end{array}$

$\begin{array}{lc}\text { Paper III } & 83\end{array}$ 


\section{CHAPTER 1}

\section{Introdution}

In 1868 the French astronomer Jules Janssen discovered a yet unknown black line in the spectrum of the light from the Sun, as can be observed e.g. by letting light pass through a prisma. A number of similar spectral lines, corresponding to the absorption of specific frequencies of visible light by some molecule or atom, were already known and understood, but this new line at $587.56 \mathrm{~nm}$ was not one of them. It was initially mistaken as an additional absorption peak of sodium, but using theoretical arguments the two Englishmen Norman Lockyer and Edward Frankland concluded that it was instead the result of a new element. Inspired by the Greek word for the Sun, helios, they named it helium. Their arguments were later shown to be correct, and in 1895 this noble gas was isolated for the first time. We now know that helium is the sixth most common gas in the atmosphere, at a volume concentration of approximately 5 parts per million.

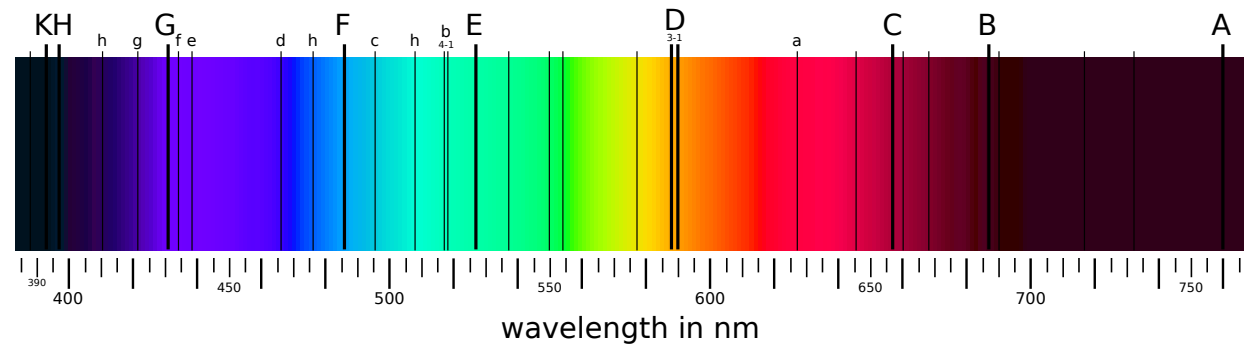

Figure 1.1. The Fraunhofer lines of the optical spectrum of the Sun. The $\mathrm{D}_{3}$ feature at $587.56 \mathrm{~nm}$ is the helium absorption line first discovered in 1868. Public domain picture. 
This is thus an example in which the measurement of the optical properties of a material in combination with theory resulted in the discovery of a new material, and even a new element at that. Additionally, the element was discovered at a staggeringly $\sim 1.5 \times 10^{11} \mathrm{~m}$ distance, and it took as much as 27 years before it could first be isolated on Earth.

This licentiate thesis deals with the development of theoretical methods by which we can understand the molecular properties of a material. These methods can be combined with experimental measurements in a manner similar to the above example in order to reach a fundamental understanding of the characteristics of the material. Alteratively, such theoretical treatment can be used to guide the development of new molecular materials with some desired properties. In this thesis, the focus is the phenomena of absorption of X-ray radiation, and in order to understand this property we utilize the theoretical framework of quantum chemistry.

\section{Quantum chemistry}

Intersecting physics and chemistry, quantum chemistry is a field of science in which we seek to understand the behaviour of matter at a molecular scale. In order to reach this understanding, it is often necessary to use a quantum mechanical description of at least the electrons of the molecule, as they cannot be well described by classical mechanics. For even greater validity, it is sometimes necessary to include also relativistic effects, both as the potential exhorted on tightly bound electrons is large enought so that scalar relativistic effects becomes important, and as spin-orbit couplings are influencial (or even vital) for the proper description of a number of chemical properties.

In this thesis, we focus on wave function-based approaches by which the electronic structure of the molecules can be modeled, accounting explicitly for the quantum mechanical behaviour of the electrons by the construction of electronic wave functions. Amongst these approaches, the hierachy of coupled cluster methods offers some of the most accurate quantum chemical methods available to date. It is thus desirable to utilize this electronic structure method for understanding molecular properties, such as X-ray absorption processes, and this thesis deals with the development of a scheme by which such properties can be calculated.

In order to understand the interaction of electromagnetic radiation and molecules, electronic response theory offers a general formulation in which a plethora of interactions can be understood. In this framework, the interaction is studied by use of perturbation theory, yielding time-dependent molecular properties which can be used to obtain a detailed understanding the physical processes, and combined with experimental studies we have a powerful tool by use of which materials can be characterized at the molecular level.

As a historical perspective, consider the following quite well-known quote:

The underlying physical laws necessary for the mathematical theory of a large part of physics and the whole of chemistry are thus completely known, and the difficulty is only that the exact application of these laws leads to equations much too complicated to be soluble. It 
therefore becomes desirable that approximate practical methods of applying quantum mechanics should be developed, which can lead to an explanation of the main features of complex atomic systems without too much computation.

$$
\text { P. A. M. Dirac, } 1929 \text { [1] }
$$

This statement has hitherto stood the test of time, and it is the development of the 'approximate practical methods of applying quantum mechanics' that is the objective of this thesis. With the simultaneous development of such methods and increase in computational power by means of high-performance computing, the size of the systems and complexity of the problems that are viable in the future are yet to be discovered.

\section{X-ray spectroscopies}

The different spectroscopies using X-ray radiation are fields of science that have benefited notably by quite recent techological innovations, resulting in highly advanced synchrotron radiation facilities for which large radiation intensities and frequency accuracies can be met. Further, advances in the development of X-ray free electron lasers offers a new field of science that is yet relatively unexplored.

For the purposes of this thesis, we are interested in the absorption of photons of an energy below that of the core ionization potentials of the molecular samples. By tuning the frequency, it is possible to get a very local probe that studies the nearest environment of specific atoms, by exciting the core electrons to bound excited states. This tool is thus very sensitive to the environment, surface structure, chemical composition and other aspects can be studied in a reliable manner. The experimental technique outlined here is an example of X-ray absorption spectroscopy (XAS), more precisely in the near-edge X-ray absorption fine structure (NEXAFS) region. By increasing the photon energy to values $20-30 \mathrm{eV}$ above the ionization threshold of the targeted elements, we get also the extended $X$-ray absorption fine structure (EXAFS) [2]. In this region the studied core electrons are excited to the continuum and the features that are seen are a result of the interaction between the photoelectrons and the environment, resulting in constructive or destructive interference which results in increases or decreases in the absorption cross-section [3]. Together, NEXAFS and EXAFS forms the X-ray absorption fine structure (XAFS), for which a prototypical spectrum can be found in Fig. 1.

In addition to the absorption processes described above, X-ray radiation can interact with molecules in a number of additional manners, two of which will be briefly discussed here. The radiation can be used to identify core electron binding energies by X-ray photoelectron spectroscopy (XPS), in which core electrons are excited into the continuum and measurements of the kinetic energies and angular distributions are used to characterize the sample. Additinally, the de-excitation of valence eletrons into core states can be studied in terms of emission of X-ray radiation, yielding X-ray emission spectroscopy (XES).

Before closing this discussion of X-ray absorption processes, it is worth mentioning the fate of an irradiated molecule that have undergone core excitation. Focus on XAS in the NEXAFS region, for which the excited electron is initially 
bound. As this excited state is inherently unstable, the core hole will be filled by a valence electron quickly after the excitation. The change in potential energy of this de-excited electrons must then be released by some means, and if this superseed the binding energy of another valence electron, this electron can be emitted. The emitted electron is in this case an Auger electron, carrying away any additional energy as kinetic energy. The emission of this Auger electron results in a valence hole, which a higher-lying valence electron can fill and the remaining energy may yet again be carried away with an Auger electron. As a result of these free Auger electrons and series of de-excitation processes, X-ray radiation can easily destroy the studied sample.

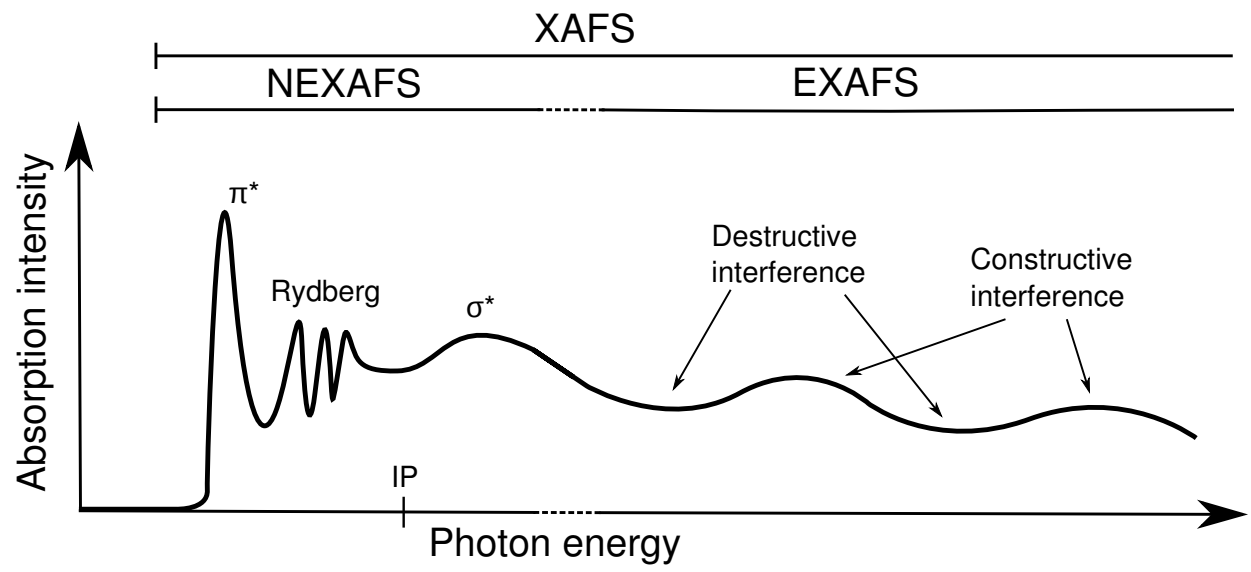

Figure 1.2. Prototypical X-ray absorption spectrum, with an intense $\pi^{*}$ transition lowest in energy, weak Rydberg resonances next and broader $\sigma^{*}$ features above the ionization potential $[3,4]$. At $20-30 \mathrm{eV}$ the near-edge X-ray fine structure region mix with the extended X-ray fine structure, in which scattering of the photoelectrons by the its environment gives features corresponding to constructive and destructive interference [2].

\section{Remark}

The material presented in this licentiate thesis have in parts been reused from an earlier Master of Science thesis written by the author [5], adapted where appropriate. 


\section{CHAPTER 2}

\section{Electronic structure theory}

In quantum chemistry, there exists a plethora of approaches by which a molecular system can be considered, with the most appropriate choice in any given situation depending entirely on e.g. the size of the system, the desired accuracy and the properties of interest. For the purpose of this thesis, we will focus on methods based on the electronic wave function. The reason for this is that we are interested in properties depending on the response of the electronic structure of the molecular systems, and we approach this in a wave function framework as this offers a reliable, hierarchical method of approaching the correct physical description. Amongst the wave function-based methods, focus will be on the coupled cluster approach, which include some of the most accurate quantum chemical methods available to date.

In this chapter, we first consider some fundamental aspects of wave functionbased electronic structure theory, methods of constructing many-electron wave functions, the single-electron description, and the effects of relativity on molecular systems. Following this, we continue with general coupled cluster theory, truncated and some by other means approximate coupled cluster theory, and the chapter includes an illustrative numerical example.

This chapter is written with the assumption that the reader has some familiarity with the general theory of quantum chemistry, as can be found in e.g. Refs. [6$9]$. 


\subsection{Fundamentals of electronic structure theory}

\subsubsection{Ab initio electron wave function methods}

For the description of any quantum mechanical system, the wave function satisfy the time-dependent Schrödinger equation

$$
i \hbar \frac{\partial}{\partial t}|\Psi\rangle=\hat{H}|\Psi\rangle \text {. }
$$

In the case of an unperturbed molecular system consisting of $N$ electrons and $M$ nuclei, the non-relativistic molecular Hamiltonian can be expressed as

$$
\hat{H}=-\sum_{i=1}^{N} \frac{1}{2} \nabla_{i}^{2}-\sum_{A=1}^{M} \frac{1}{2 M_{A}} \nabla_{A}^{2}-\sum_{i=1}^{N} \sum_{A=1}^{M} \frac{Z_{A}}{r_{i A}}+\sum_{i=1}^{N} \sum_{j>i}^{N} \frac{1}{r_{i j}}+\sum_{A=1}^{M} \sum_{B>A}^{M} \frac{Z_{A} Z_{B}}{R_{A B}},
$$

using atomic units, for simplicity. In this expression, the first two terms describe the kinetic energy of the electrons and nuclei, and the last three the Coulombic interaction between the electrons and nuclei, electrons and electrons, and nuclei and nuclei, respectively.

Due to the large difference in mass between the electrons and nuclei, we separate these entities by the Born-Oppenheimer approximation, resulting in an electronic Hamiltonian where the two terms treating only nuclei contribute with a constant. For the purposes of this thesis, this approximation is reasonable, leading only to a neglible error and offering a substantial simplification of the problems at hand. However, it is clear that the resulting electronic Hamiltonian still yields a many-particle problem that cannot be solved analytically (except in the trivial case of one or two electrons), and numerical schemes are thus necessary.

As a first approximation, the electron-electron interaction can be modelled as an interaction between single electrons and the mean-field of the other electrons. This corresponds to the Hartree-Fock (HF) approximation and yields results that capture the main contributions to the electronic energy. The approach requires no parametrization from experiment or other calculations, and is thus an example of a ab initio wave function method. However, as the electrons interacts in manners that cannot be described by a mean-field approximation, the HF method lacks important electron correlation, and the retrieval of this constitutes one of the major issues in quantum chemistry. This correlation can be subdivided into two different effects, static and dynamic correlation.

Static correlation is important when a molecule requires several (nearly) degenerate determinants for a good description of the ground state. Cases when this becomes important includes e.g. bond breaking and quasi-degenerate ground states with low lying excited states. In order to capture these effects, multi-reference methods are necessary. As the response method discussed in this thesis is developed for single-reference coupled cluster, static correlation will not be considered and all correlation discussed is hereby understood to mean dynamic correlation.

Dynamic correlation arise from the correlation in the movement of the electrons, resulting in a correlation energy that can be given as

$$
E_{\mathrm{corr}}=E_{0}-E_{\mathrm{HF}} \text {. }
$$


Due to the variational condition, the HF energy $\left(\mathrm{E}_{\mathrm{HF}}\right)$ is always larger than the exact energy $\left(\mathrm{E}_{0}\right)$, and the correlation energy is thus negative. Several post$H F$ methods have been developed, in which the HF wave function is taken as a a reference, and the correlation is included by some means to create a more physically correct wave function.

One such method is configuration interaction (CI), in which correlation is accounted for by means of exciting the electrons in the HF (or possibly some other reference) state to virtual orbitals, forming a CI wave function as a sum of the reference determinant, all singly excited determinants, all doubly excited determinants and so on. This is done variationally, and if the CI excitation space is included up to excited determinants of order $N$ (i.e. the number of electrons in the system), the resulting wave function is exact (under the restriction of the other approximations, e.g. the Born-Oppenheimer approximation). However, constructing this Full CI (FCI) wave function is unfeasible for most systems, and the CI excitation space is truncated by necessity. Unfortunately, owing to the linear nature of the CI expansion, the wave function resulting from such a truncation is no longer size-consistent, meaning that the energy of two infinitely separate molecules is different from that of the sum of the energies of the two molecules.

A related methodology is that of coupled cluster (CC), for which a non-linear expansion of excited determinants is used to form the $\mathrm{CC}$ wave function, thus exhibiting size-consistency. The method is hierarhical in the inclusion of electron correlation and stands out as one of the most accurate approaches in quantum chemistry. As for negative aspects, it is non-variational in most implementations and very computationally demanding, but these concerns will be discussed in more detail in next section.

The electronic structure methods discussed thus far forms the approaches by which the $N$-electron wave function is formed, but in order to do so we need a set of trial wave functions, or basis functions to act upon. Chosing the appropriate basis sets corresponds then to the one-electron description, and this choice needs to be balanced against the electronic structure method. Especially for calculated calculations, such as for CC, the basis set needs to be sufficiently flexible so that the wave function can be correlated properly with virtual excitations. In this thesis we have utilized the correlation-consistent polarized valence $X$-zeta (ccpVXZ) basis sets developed by Dunning and coworkers [10]. To better describe the core relaxation these basis sets have been augmented with core-polarizing functions [11], and additional augmentation using diffuse functions have been used to better describe the excited states [12]. Finally, as many of the excited states that are studied in here is of a Rydberg character, we have adopted the proposition of Kaufmann et al. [13] and supplemented the basis sets with Rydberg functions.

\subsubsection{Relativistic effects}

We have so far only considered the non-relativistic realm of quantum chemistry, assuming that relativistic effects are small. However, this is not always the case, and for a proper description of the spin- $1 / 2$ four-component relativistic wave function 
must satisfy the Dirac equation, given in the form

$$
i \hbar \frac{\partial}{\partial t} \Psi(\bar{r}, t)=c(\tilde{\beta} m c-i \hbar \tilde{\alpha} \cdot \nabla) \Psi(\bar{r}, t) .
$$

Here the wave function $\Psi$ is a four-component entity and $\tilde{\alpha}$ and $\tilde{\beta}$ are $4 \times 4$ matrices [14].

However, if the relativistic effects are small, it is possible to avoid solving this complex problem and effects can instead be considered in a two-component realm, or as a perturbation. As this work only considers elements from the first and second period and excitations from the $1 s$ orbitals, relativistic effects are in this case small and scalar. As a result of this, the second-order Douglas-Kroll-Hess Hamiltonian [15-17] is able to capture the majority of the relativistic effects. It is to be noted that relativity generally acts in such a manner that $s$ and $p$ orbitals contract, due to reduced experienced screening, while the remaining orbitals decontract. This means that different sets of basis sets should be used for relativistic calculations, as the standard sets are optimized for non-relativistic calculations. As will be seen, the relativistic effects in this work are all small and this is thus not necessary.

\subsubsection{Approaching the correct wave function}

As have been seen in Sections 2.1.1 and 2.1.2, the construction of the correct wave functions have three levels in which an appropiate description must be found: the construction of the $N$-electron wave function, the one-electron description and the relativistic effects. This can be visualized as in Fig. 2.1, where the correct wave function is approached by moving simultaneously along the three axes. Also, as the computational cost increase for each step along the degrees of freedom, we see that the treatment of large molecules is restricted to the volume close to origo, and we thus need schemes by which the effects of an improved description can be estimated.

Two remarks should be added in concern with this model. First, the axes with the relativistic models and the basis sets are hierarhical, meaning that moving outwards along them improves the description of the wave function. However, this is not always the case for the third axis, as some properties may be better described with a electronic structure method lower in the ordering, due to cancellation of errors or other effects. This is especially true for Kohn-Sham density functional theory (KS-DFT), for which there is no a priori way of determining which functionals are most suitable for a specific problem. There is, however, a general trend for KS-DFT in which the local density approximation (LDA) gives functionals of a lower quality than the generalized gradient approximation (GGA), which in turn is superseeded by hybrid functionals. The hybrid functionals can be further improved for response calculations by ensuring a proper long-range behavior, constructing the range-separated hybrid functionals.

As the second remark, the model may give the impression that variations along these three axes are decoupled. This is not the case, especially for the basis sets and electronic structure methods. In order to properly account for electron 
correlation the basis set needs to be sufficiently flexible. If this is not the case the results can actually be less reliable than using a more approximate electronic structure method. Further, relativistic effects and electron correlation effects are not additive, so these needs to be considered simultaneously as well.

In conclusion, the approximations on the different axes needs to be balanced in order to approach the correct wave function in a reliable, efficient manner.

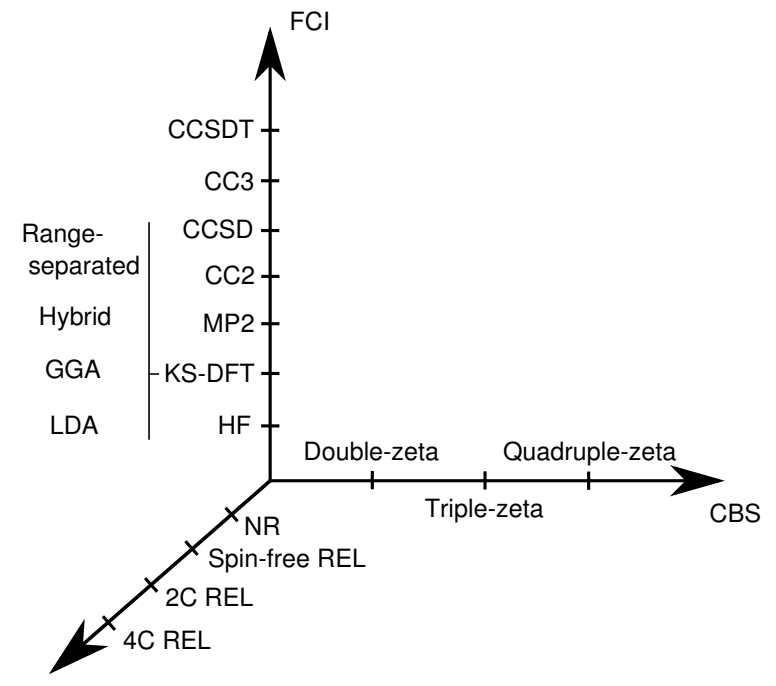

Figure 2.1. Approximate ordering of theoretical models for describing electron structure. Model partially adopted from Ref. [18].

\subsection{Coupled cluster theory}

The topic of this thesis is the development and application of response methods in the coupled cluster (CC) framework, since this contains some of the most accurate $a b$ initio electronic wave function methods in quantum chemistry, as discussed in Section 2.1.1. The methodology contains a number of desirable features, such as a controlled, hierarchical convergence towards the exact wave function. This hierarchy of CC methods thus offers a way of accounting for relaxation in a reliable manner, enabling us to determine the requirements imposed by core excitation processes.

We will now consider the fundamentals of coupled cluster theory, both in the standard formulation as well as a number of approximate CC methods developed for response theory. Calculations on the first excitation of neon will serve as an illustration of the CC hierarchy, as well as the effects of relativity and one-electron description. For more details on the theory, see e.g. Refs. $[6,8,9]$. 


\subsubsection{The exponential ansatz}

The coupled cluster approach utilizes excitations to unoccupied (virtual) orbitals in order to obtain the correlation energy, with an excitation operator

$$
\hat{T}=\hat{T}_{1}+\hat{T}_{2}+\cdots+\hat{T}_{N}
$$

for a system of $N$ electrons. The separate $\hat{T}_{i}$ designates excitation operators that simultaneously excites $i$ electrons from occupied to unoccupied orbitals.

If all possible electron excitations are included, as in Eq. 2.5, the coupled cluster method yields an exact description of the electronic many-particle wave function within any other approximation that might have been emplyed (i.e. it corresponds to full CI). However, this is computationally feasible only for very small systems and a truncation is thus employed in most applications, giving a hierarchical approach towards the exact solution. The level of this truncation determines the model in the hierarchy of CC methods: including only single excitations yields coupled cluster singles (CCS), including both single and double excitations yields coupled cluster singles and doubles [19] (CCSD), and so on. For practical purposes the inclusion of triple excitations are generally too demanding, and such effects can instead be introduced in an approximate manner $[6,9]$.

The effects of the single and double excitation operators are given as

$$
\begin{aligned}
& \hat{T}_{1}\left|\Psi_{0}\right\rangle=\sum_{i}^{\text {occ virt }} \sum_{a}^{a} t_{i}^{a} \hat{\tau}_{i}^{a}\left|\Psi_{0}\right\rangle=\sum_{i}^{\text {occ }} \sum_{a}^{\text {virt }} t_{i}^{a}\left|\Psi_{i}^{a}\right\rangle, \\
& \hat{T}_{2}\left|\Psi_{0}\right\rangle=\sum_{i>j}^{\text {occ virt }} \sum_{a>b}^{\text {vir }} t_{i j}^{a b} \hat{\tau}_{i j}^{a b}\left|\Psi_{0}\right\rangle=\sum_{i>j}^{\text {occ }} \sum_{a>b}^{\text {virt }} t_{i j}^{a b}\left|\Psi_{i j}^{a b}\right\rangle,
\end{aligned}
$$

where $t_{i}^{a}$ and $t_{i j}^{a b}$ are the expansion coefficients, known also as coupled cluster amplitudes, and $\hat{\tau}_{i}^{a}$ and $\hat{\tau}_{i j}^{a b}$ are the explicit excitation operators, most succinctly expressed using second quantization. To avoid double-counting we impose the conditions of $i>j$ (occupied orbitals) and $a>b$ (unoccupied orbitals) in Eq. 2.7.

The coupled cluster wave function is constructed by use of the exponential ansatz, where we act on a reference wave function by an exponential operator as

$$
|\mathrm{CC}\rangle=e^{\hat{T}}\left|\Psi_{0}\right\rangle=e^{\hat{T}}|0\rangle .
$$

The reference wave function is usually in the form of a single-reference HF wave function. In this case the reference state is static and the system considered is more accurately described if it is dominated by a single electronic configuration (i.e. weak static correlation) [20].

Expanding the exponential operator yields

$$
\begin{aligned}
e^{\hat{T}} & =1+\hat{T}+\frac{1}{2} \hat{T}^{2}+\ldots \\
& =1+\hat{T}_{1}+\left(\hat{T}_{2}+\frac{1}{2} \hat{T}_{1}^{2}\right)+\left(\hat{T}_{3}+\hat{T}_{2} \hat{T}_{1}+\frac{1}{6} \hat{T}_{1}^{3}\right)+\ldots
\end{aligned}
$$


reordered such that the first term gives the reference state, the second all single excited states, the third all double excited states and son on.

As can be seen, excited states of a higher order also includes simultaneous excitations of lower order, e.g. the doubly excited states includes not only the connected double excitations $\hat{T}_{2}$, but also the disconnected simultaneous single excitations $\hat{T}_{1}^{2}$. This results in coupled cluster being size-extensive, i.e., it yields consistent results for arbitrary numbers of non-interacting molecules. Further, disconnected excitations may be most important for higher-order excitations, e.g. the $\hat{T}_{2}^{2}$ terms are more important for quadruple excitations than $\hat{T}_{4}[19,21]$, so the system can be well described at a low order of truncation.

The coupled cluster ground state can in principle be solved by use of the variational principle, but this is only feasible for small systems due to the nonlinear nature of the CC ansatz. The procedure by which CC is more commonly considered is by projecting the Schrödinger equation onto a HF state $|0\rangle$ and a manifold of excited states

$$
\hat{\tau}_{n}|0\rangle=|n\rangle .
$$

This manifold consists of all single excited states for CCS, all single and double excited states for CCSD and so on.

For convenience, we choose to multiply the time-independent Schrödinger equation by the exponential operator $e^{-\hat{T}}$ from the left, resulting in what can be regarded as an effective, non-Hermitian Hamiltonian

$$
\hat{H}_{T}=e^{-\hat{T}} \hat{H} e^{\hat{T}}
$$

called the similarity-transformed Hamiltonian. The Schrödinger equation becomes

$$
\hat{H}_{T}|0\rangle=E|0\rangle \text {. }
$$

By projection onto the HF state and the manifold of excited states this yields

$$
\begin{aligned}
& \left\langle 0\left|\hat{H}_{T}\right| 0\right\rangle=E, \\
& \left\langle n\left|\hat{H}_{T}\right| 0\right\rangle=0,
\end{aligned}
$$

which corresponds to the CC equations and can be solved for the CC ground state energy and amplitudes. Further, from Eq. 2.13 it can be shown that

$$
\langle 0| e^{-\hat{T}}=\langle 0|
$$

as all excitation operators here attempts to de-excite a reference ground state and thus do not contribute. If the reference state used is an optimized HF state, it can be shown that one-particle excitation operators does not contribute to the electronic energy in a connected manner, as following Brillouin's theorem [6]

$$
\left\langle 0\left|\hat{H} \hat{T}_{1}\right| 0\right\rangle=0 .
$$


Using Eqs. 2.9, 2.13, 2.15, and 2.16, and the fact that the Hamiltonian is a twoparticle operator, the $\mathrm{CC}$ energy expression is simplified as

$$
\begin{aligned}
E & =\left\langle 0\left|\hat{H}\left(1+\hat{T}+\frac{1}{2} \hat{T}^{2}+\ldots\right)\right| 0\right\rangle \\
& =\left\langle 0\left|\hat{H}\left(1+\hat{T}_{2}+\frac{1}{2} \hat{T}_{1}^{2}\right)\right| 0\right\rangle,
\end{aligned}
$$

as the higher-order excitation operators yields states that are orthogonal to the reference state. The energy of a CC method thus depends explicitly on only the single and double excitations, while higher-order excitations contribute implicitly in the coupling of amplitudes, as given by Eq. 2.14.

\subsubsection{Approximate coupled cluster methods}

As discussed in connection with Eq. 2.5, considering all possible excitations is computationally unfeasible for most systems, and some way of decreasing the size of the excitation manifold needs to be employed. The most direct way of doing it has already been treated, i.e., truncating the excitation operator to certain order, but for the inclusion of higher-order excitation this may still be too demanding, with e.g. CCSDT scaling as $m^{8}$ ( $m$ being the number of basis functions). A major topic in CC theory is thus the development of methods by which approximate higherorder excitations can be included at a lower computational cost, and the work presented in this thesis has utilized two such methods: the approximate methods for inclusion of double excitations CC2 [22] and triple excitations CCSDR(3) [23], both developed for the calculation of molecular properties.

The CC2 method utilizes perturbation theory to determine the order of contribution from the different excitations. As an illustration for ground state energy: split the manifold of excited states into one manifold for single excitation $\left(\left|n_{1}\right\rangle\right)$ and one for the double excitation $\left(\left|n_{2}\right\rangle\right)$. The CCSD amplitude equations for single and double excitations (starting from Eq. 2.14) can then be separated, and, using the Baker-Campbell-Hausdorff expansion [6], we get

$$
\begin{aligned}
& 0=\left\langle n_{1}\left|\hat{H}_{T}+\left[\hat{H}_{T}, \hat{T}_{2}\right]\right| 0\right\rangle, \\
& 0=\left\langle n_{2}\left|\hat{H}_{T}+\left[\hat{H}_{T}, \hat{T}_{2}\right]+\frac{1}{2}\left[\left[\hat{H}_{T}, \hat{T}_{2}\right], \hat{T}_{2}\right]\right| 0\right\rangle .
\end{aligned}
$$

The single excitation manifold amplitude equation is kept the same, while the double excitation manifold amplitude equation only retains contributions of lowest non-vanishing order, so that the last term in this equation is disregarded. What is obtained is a subset of the CCSD equations, solved iteratively with a scaling of $m^{5}$.

A similar treatment of triple excitation contributions has been developed, designated CC3 [24], and this yields an iterative method of a scaling of $\mathrm{m}^{7}$, and is thus still computationally demanding. It is instead possible to define a perturbative correction to the CCSD excitation energies that is of same order of accuracy as CC3, but at a lower computational cost. This corresponds to the CCSDR(3) method, 
which is a non-iterative correction to CCSD eigenvectors that incorporates lowest order triple corrections.

With the perturbative-theoretical treatment it is possible to determine the order to which these approaches are correct for e.g. ground state and property calculations [25]. Such order analysis is given in Table 2.1 for the ground state and some excitation energies along with the full scaling of the different methods, save for CCSDR(3). For practical purposes, benchmark studies on valence excitations have shown that CCSDR(3) gives results close to those obtained by CC3 [26], and is thus a cost-efficient alternative. Further, basis set effects on CC2 and CCSDR(3) are seem to be similar [27], and CC2 may perform better than CCSD for certain systems [28].

Table 2.1. Perturbative-theoretical analysis of the order to which a selection of coupled cluster methods are correct for calculation of ground state energies $\left(E_{0}\right)$, single and double electron excitation energies (SE and DE, respectively), as well as the scaling of the methods.

\begin{tabular}{lcccc}
\hline \hline Method & $E_{0}$ & $E_{s}$ & $E_{D}$ & Scaling \\
\hline CCS & 1 & 1 & - & $m^{4}$ \\
CC2 & 2 & 2 & 0 & $m^{5}$ \\
CCSD & 3 & 2 & 1 & $m^{6}$ \\
CC3 & 4 & 3 & 2 & $m^{7}$ \\
CCSDT & 4 & 4 & 2 & $m^{8}$ \\
\hline \hline
\end{tabular}

\subsubsection{Illustrative calculations on neon}

As an illustration of the performance of the $\mathrm{CC}$ hierarchy, the total electronic ground state and first excitation $\left(\mathrm{X}^{1} S_{0} \rightarrow 1^{1} P_{1}^{0}\right)$ energies of neon using different CC models are shown in Fig. 2.2. The excitation energy has been measured to be $16.848 \mathrm{eV}$ [29]. Further, to account for all three dimensions of the model given in Fig. 2.1 and discussed in Section 2.1.3, different basis sets have been employed and scalar relativistic effects have been addressed using the second order spin-free Douglas-Kroll-Hess Hamiltonian for CCS and CC3 calculations (only the latter is shown in the figure).

As discussed in Section 2.1.3, we first observe that the three dimensions of Fig. 2.2 cannot be considered as decoupled. Increasing the basis set from d-aug-ccpVDZ to t-aug-cc-pVTZ results in a decrease in ground state energy of $1.00 \mathrm{eV}$ for CCS, but $3.06 \mathrm{eV}$ for CCSD, illustrating that a larger basis set is more important for more electron correlated calculations, as more flexibility is needed for the virtual excitations to correlate the system. Meanwhile, adding scalar relativistic effects to the calculation of CCS ground state energy decrease the energy by $3.41 \mathrm{eV}$, while corresponding value for CC3 is $3.42 \mathrm{eV}$.

Consider the convergence towards the experimental value as given by the $\mathrm{CC}$ hierarchy using a t-aug-cc-pVTZ basis set. The ground state energy decrease from CCS to CC2, but then increase again for the CCSD calculation, illustrating the non-variational nature of the $\mathrm{CC}$ method. A variational method should decrease 
monotonous as more electron correlation is addressed. Following CCSD the energy decreases in all cases when the effects of triple excitations are approximately accounted for. For the excitation energy, we see a large difference between the CCS result and all the other results, where adding only double excitations overestimates the correction (this overestimation is larger for the approximate treatment of double excitations, i.e. CC2), and triple excitations corrects for this somewhat. This large discrepancy for CCS can be partially understood as an effect of the lack of relaxation effects, a subject that will be treated thoroughly later in this thesis.

The best theoretical results included in this illustration, obtained from a scalar relativistic calculation at a CC3/decontracted p-aug-cc-pV6Z level, yields an excitation energy of $16.781 \mathrm{eV}$, this being $0.067 \mathrm{eV}$ too low comparing to the experimental value [29]. The remaining discrepancy is thus small and likely an effect of insufficient correlation and relativistic treatment.

In conclusion, it is seen that the $\mathrm{CC}$ method has a relatively high demand in terms of basis sets used, but a triple- $\zeta$ basis set seems to be reasonably well balanced with CCSD. Including double excitation gives a substantial improvement of energetics, and triple excitations can be included either by using CC3, or more cheaply by a CCSDR(3) correction of CCSD energetics. Relativistic effects are seen to contribute mainly with an absolute shift in energy for this illustration.

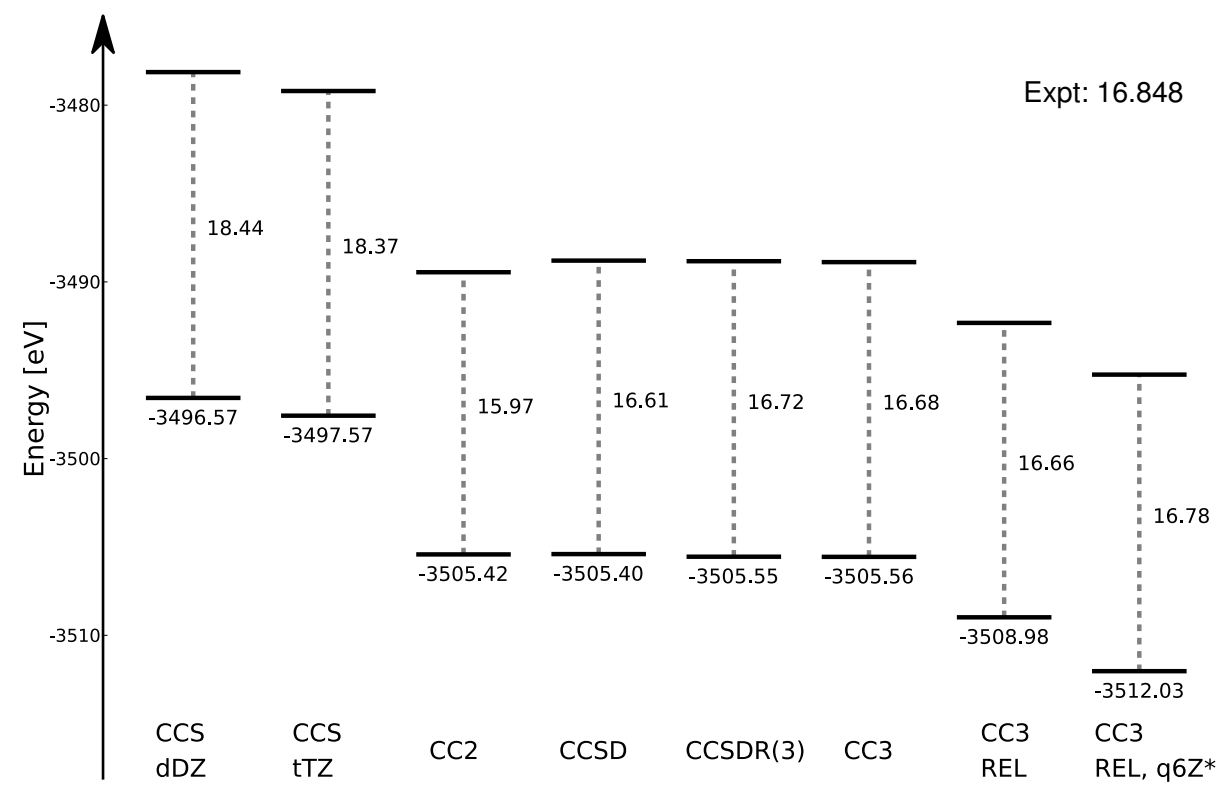

Figure 2.2. Theoretical ground state and $\mathrm{X}^{1} S_{0} \rightarrow 1^{1} P_{1}^{0}$ excitation energies of neon, as obtained with a number of CC methods. The basis set abbreviations are for d-aug-ccpVDZ, t-aug-cc-pVTZ, and a decontracted p-aug-cc-p6Z basis, and t-aug-cc-pVTZ is used if nothing else is stated. REL designates that scalar relativistic effects has been addressed by use of the second order spin-free Douglas-Kroll-Hess Hamiltonian. Experimental excitation energy from Ref. [29]. 


\section{CHAPTER 3}

\section{Molecular response theory}

In the field of theoretical spectroscopy, response theory offers a framework general enough to be used for a plethora of different spectroscopies, seemingly too different in nature to be treated on common grounds. In this approach time-dependent perturbation theory is used for approximate state electronic structure theory. The molecular properties, which describe the interaction of the molecule with an internal or external perturbation, are determined as the response of the electronic wave function (or electron density). For the purposes of this thesis, the perturbation will from this point on be assumed to be external.

In this chapter, we will discuss the fundamentals of response theory for exact states and determine the linear response function that is the key property needed for the calculation of X-ray absorption spectra. We then continue with the derivation of the damped coupled cluster linear response function, for which two different computational schemes are presented.

For a thorough investigation on the current status on (wave function-based) response theory, we refer to the comprehensive work of Helgaker et al. [30], in which an extensive reference list can guide the reader to details concerning any molecular properties of interest.

\subsection{Exact state response theory}

First, let us consider the case of exact state response theory, where the exact eigenstates of the Hamiltonian of the unperturbed system is known. For this case, we discuss the general determination of molecular properties and the damped formulation of response theory using which it is possible to address external perturbations exciting the molecular system to some excited state. The determination of response functions using the density matrix and the quasi-energy formalism [31] will 
also be presented.

\subsubsection{General response theory}

When an external field is applied to a molecular system the system interacts with this field and responds to the applied perturbation. If such field is of sufficiently small field strength, i.e. sufficiently small intensity, we can address the response using perturbation theory with the perturbed Hamiltonian

$$
\hat{H}(t)=\hat{H}_{0}+\hat{V}(t),
$$

where is $\hat{H}_{0}$ the unperturbed Hamiltonian and $\hat{V}(t)$ a homogeneous, external field [32]. The requirement of a weak perturbation is met for most externally applied fields, as even a macroscopically strong field, e.g. a magnetic field of 10 Tesla, will be very weak as compared to the internal fields of the molecule and amount only to circa $4.3 \times 10^{-6}$ atomic units.

If the perturbing field is taken as a non-oscillating, static field, i.e. $\hat{V}(t)=\hat{V}_{\alpha} F_{\alpha}$ for a field direction $\alpha$, the problem is reduced to the time-independent case. The time-independent Schrödinger equation can then be utilized in order to obtain ground and excited state energies that are well-defined. This is to be compared with the case of time-dependent external fields, where the interaction between the field and the molecular system yields ill-defined energies. The energy of the static case can be considered with a Taylor expansion at zero perturbing field strength

$$
E=E_{0}+\left.F_{\alpha} \frac{\partial E}{\partial F_{\alpha}}\right|_{F_{\alpha}=0}+\left.F_{\alpha} F_{\beta} \frac{1}{2} \frac{\partial^{2} E}{\partial F_{\alpha} \partial F_{\beta}}\right|_{F_{\alpha}=F_{\beta}=0}+\ldots,
$$

with implied summation over indices. Molecular properties are now identified as field derivatives of different orders and different perturbing fields, as the observables associated with the properties changes according to these derivatives. For example: the polarizability may be obtained through the second derivative of the energy with respect to a perturbing electric field, while the first derivatives corresponds to the permanent dipole moment, both at zero field strength. If the field is time-dependent, and the energy thus undefined, other methods must be utilized, as will now be discussed.

Consider a field that is periodic at the time of interest. The form of the perturbation operator is chosen as

$$
\hat{V}_{\alpha}(t)=\sum_{\omega} \hat{V}_{\alpha}^{\omega} F_{\alpha}^{\omega} e^{-i \omega t} e^{\epsilon t}
$$

where $F_{\alpha}^{\omega}$ is the Fourier amplitude of the field along a molecular axis $\alpha$. A small, positive $\epsilon$ gives the adiabatical switch on as $t \rightarrow-\infty$, while ensuring that the last term differs negligible from unity at times close to $t=0$ (i.e. present time). This condition is imposed to ensure that the system resided in the ground state at a time long past, sufficiently long ago so that all memory of this process is lost. The summation is understood to be over both positive and negative frequencies, and 
due to the real nature of the perturbation we have $F_{\alpha}^{-\omega}=\left[F_{\alpha}^{-\omega}\right]^{*}$ and $\hat{V}_{\alpha}^{-\omega}=$ $\left[\hat{V}_{\alpha}^{-\omega}\right]^{\dagger}=\hat{V}_{\alpha}^{\omega}$.

The time-dependent molecular properties can now be defined through the timedependent expectation value of an observable, associated with an operator $\hat{\Omega}$. We first expand the wave function in orders of the perturbation

$$
|\psi(t)\rangle=\left|\psi^{(0)}\right\rangle+\left|\psi^{(1)}\right\rangle+\left|\psi^{(2)}\right\rangle+\ldots
$$

and the expectation value of the operator is expanded as

$$
\begin{aligned}
\langle\psi(t)|\hat{\Omega}| \psi(t)\rangle= & \left\langle\psi^{(0)}|\hat{\Omega}| \psi^{(0)}\right\rangle \\
& +\left\langle\psi^{(1)}|\hat{\Omega}| \psi^{(0)}\right\rangle+\left\langle\psi^{(0)}|\hat{\Omega}| \psi^{(1)}\right\rangle \\
& +\left\langle\psi^{(2)}|\hat{\Omega}| \psi^{(0)}\right\rangle+\left\langle\psi^{(1)}|\hat{\Omega}| \psi^{(1)}\right\rangle+\left\langle\psi^{(0)}|\hat{\Omega}| \psi^{(2)}\right\rangle \\
& +\ldots,
\end{aligned}
$$

rewritten for simplicity

$$
\langle\psi(t)|\hat{\Omega}| \psi(t)\rangle=\left\langle\hat{\Omega}^{(0)}\right\rangle+\left\langle\hat{\Omega}^{(1)}\right\rangle+\left\langle\hat{\Omega}^{(2)}\right\rangle+\ldots,
$$

where the different terms at the right-hand-side are again understood as the correction of the expectation value of a given order. By use of the perturbation operator in Eq. 3.3, the expansion is written as

$$
\begin{aligned}
\langle\psi(t)|\hat{\Omega}| \psi(t)\rangle= & \langle 0|\hat{\Omega}| 0\rangle \\
& +\sum_{\omega_{1}}\left\langle\left\langle\hat{\Omega} ; \hat{V}_{\beta}^{\omega_{1}}\right\rangle\right\rangle F_{\beta}^{\omega_{1}} e^{-i \omega_{1} t} e^{\epsilon t} \\
& +\frac{1}{2} \sum_{\omega_{1} \omega_{2}}\left\langle\left\langle\hat{\Omega} ; \hat{V}_{\beta}^{\omega_{1}}, \hat{V}_{\gamma}^{\omega_{2}}\right\rangle\right\rangle F_{\beta}^{\omega_{1}} F_{\gamma}^{\omega_{2}} e^{-i\left(\omega_{1}+\omega_{2}\right) t} e^{2 \epsilon t} \\
& +\ldots
\end{aligned}
$$

explicitly giving the expressions for the first-, second- and third-order properties. The term $\left\langle\left\langle\hat{\Omega} ; \hat{V}_{\beta}^{\omega_{1}}\right\rangle\right\rangle$ in the second-order property is refered to the linear response function, collecting all expectation values that are linear in the the perturbation. In an analogous manner, $\left\langle\left\langle\hat{\Omega} ; \hat{V}_{\beta}^{\omega_{1}}, \hat{V}_{\gamma}^{\omega_{2}}\right\rangle\right\rangle$ collects all first-order non-linear terms and are thus designated as the first-order non-linear response function (but secondorder response function), and so on for higher-order terms.

\subsubsection{Damped linear response theory}

Determining the explicit expressions for the linear and non-linear response functions can be done in several different manners, the most common being using either the Ehrenfest expectation value of the quasi-energy derivative formulation $[18,31]$. Here, we will instead focus on an approach employing the Liouville equation, but some aspects of the quasi-energy formalism will be considered in the next section in order to facilitate our discussion of coupled cluster response theory. 
Before moving to the derivation of the linear response function, there is a yet unaddressed issue of resonance frequencies in the external, perturbing field. As will be demonstrated in this section, the standard formulation of response theory suffers from divergences at resonance frequencies, due to singularities in the response equations. In order to obtain resonance-convergent expressions, we instead move to a damped formulation of response theory, by which the divergences can be accounted for, if we first consider the ways by which the molecular system relaxes and experience de-excitations.

In the absence of any external interactions (external fields, other molecules, etc.) an excited state will eventually relax into the ground state - the excited state has a finite lifetime and experiences spontaneous relaxation. If the system is allowed to interact with other molecules, e.g. by collisions, the lifetime may be lowered as the system can be relaxed through radiative and non-radiative relaxation channels. A (weak) perturbing field affecting a ground state, on the other hand, can both influence the population of the different states of the system by absorption and stimulated emission. Accounting for all these effects, the decay rates of the excited states will be such that the population of the excited states remains small and is thus possible to apply perturbation theory on the system. However, incorporating these relaxation effects directly into the Schrödinger equation is not easily done, and the effects will instead be incorporated in a phenomenological manner.

A formalism suitable for this task is the density matrix formalism. Introducing the density operator

$$
\hat{\rho}=\sum_{s} p(s)\left|\psi_{s}(t)\right\rangle\left\langle\psi_{s}(t)\right|,
$$

for the statistical ensemble of system configurations $s$ with corresponding probabilities $p(s)$. For simplicity, let us consider ensembles that can be described by a single wave function, i.e. pure states, and a wave function expansion in form of projections:

$$
\left|\psi_{s}(t)\right\rangle=\sum_{n} c_{n}^{s}(t)|n\rangle
$$

for which the matrix elements of the density operator are expressed as

$$
\rho_{m n}=\sum_{s} p(s) c_{m}^{s} c_{n}^{s *}
$$

With the Schrödinger equation 2.1 for bra- and ket-vectors, it is easy to show that the equation-of-motion for the density operator can be written as

$$
\frac{\partial}{\partial t} \hat{\rho}=\frac{1}{i \hbar}[\hat{H}, \hat{\rho}] \text {. }
$$

This equation is known as the Liouville equation. It can easily be modified to, phenomenologically, include relaxations of the system. For a matrix element of the density operator, this modification is made such that the Liouville equation becomes the damped counterpart

$$
\frac{\partial}{\partial t} \rho_{m n}=\frac{1}{i \hbar}[\hat{H}, \hat{\rho}]_{m n}-\gamma_{m n}\left(\rho_{m n}-\rho_{m n}^{\mathrm{eq}}\right),
$$


where the damping term $\gamma_{m n}$ corresponds to the rate of $\rho_{m n}$ decaying into the equilibrium value $\rho_{m n}^{\mathrm{eq}}$. We further assume that electronic excitations by thermal or other effects are negligible, and the equilibrium value is thus the ground state with matrix elements $\delta_{n 0} \delta_{m 0}$.

As the diagonal elements of the density operator can, for a pure state, be regarded as the population of the different states, the diagonal elements of the damping matrix can be understood as the inverse of the average lifetime. With this in mind, we now have a way by which the lifetimes, obtained by experiment or other means, can be phenomenologically addressed for the system.

In order to get the linear response function, the expansion of an expectation value as given in Eq. 3.5 may be considered. In the density matrix formalism expectation values are given as the trace

$$
\langle\hat{\Omega}\rangle=\operatorname{Tr}(\hat{\rho} \hat{\Omega})=\operatorname{Tr}(\hat{\Omega} \hat{\rho}),
$$

and it is thus possible to calculate the molecular properties by using an expansion of the density operator

$$
\rho_{m n}(t)=\rho_{m n}^{(0)}+\rho_{m n}^{(1)}+\rho_{m n}^{(2)}+\ldots,
$$

with a zeroth-order value given by the ground state, i.e. of the unperturbed system

$$
\rho_{m n}^{(0)}=\delta_{n 0} \delta_{m 0}
$$

With the total Hamiltonian from Eq. 3.1, the unperturbed part yields, for the equation-of-motion

$$
\left[\hat{H}_{0}, \hat{\rho}\right]_{m n}=\hbar \omega_{m n} \rho_{m n}
$$

and the damped Liouville equation 3.12 is solved in order $N$ by time integration

$$
\rho_{m n}^{(N)}=e^{-\left(i \omega_{m n}+\gamma_{m n}\right) t} \int_{-\infty}^{t} \frac{1}{i \hbar}\left[\hat{V}, \hat{\rho}^{(N-1)}\right]_{m n} e^{\left(i \omega+\gamma_{m n} t^{\prime}\right)} d t^{\prime} .
$$

The perturbation operator $\hat{V}$ is given in Eq. 3.3, and we obtain the first-order response as

$$
\begin{aligned}
\rho_{m n}^{(1)} & =\frac{1}{i \hbar} \sum_{\omega} \frac{\left[\hat{V}_{\beta}^{\omega}, \rho^{(0)}\right]_{m n} F_{\beta}^{\omega}}{i \omega_{m n}-i \omega+\gamma_{m n}+\epsilon} e^{-i \omega t} e^{\epsilon t} \\
& =\frac{1}{i \hbar} \sum_{\omega}\left[\frac{\left\langle m\left|\hat{V}_{\beta}^{\omega}\right| 0\right\rangle \delta_{n 0}}{i \omega_{m 0}-i \omega+\gamma_{m 0}+\epsilon}-\frac{\left\langle 0\left|\hat{V}_{\beta}^{\omega}\right| n\right\rangle \delta_{m 0}}{-i \omega_{n 0}-i \omega+\gamma_{n 0}+\epsilon}\right] F_{\beta}^{\omega} e^{-i \omega t} e^{\epsilon t} .
\end{aligned}
$$

Not that the frequency $\omega$ runs over both positive and negative values. The firstorder correction of the expectation value of an operator can now be identified as

$$
\begin{aligned}
\left\langle\hat{\Omega}^{(1)}\right\rangle & =\operatorname{Tr}\left(\hat{\rho}^{(1)} \hat{\Omega}\right)=\sum_{m n} \rho_{m n}^{(1)} \Omega_{m n} \\
& =-\frac{1}{\hbar} \sum_{\omega}\left[\frac{\langle 0|\hat{\Omega}| n\rangle\left\langle n\left|\hat{V}_{\beta}^{\omega}\right| n\right\rangle}{\omega_{n 0}-\omega-i \gamma_{n 0}-i \epsilon}+\frac{\left\langle 0\left|\hat{V}_{\beta}^{\omega}\right| n\right\rangle\langle n|\hat{\Omega}| 0\rangle}{\omega_{n 0}+\omega+i \gamma_{n 0}+i \epsilon}\right] F_{\beta}^{\omega} e^{-i \omega t} e^{\epsilon t} .
\end{aligned}
$$


This expression still contains the small, positive $\epsilon$, adapted for an adiabatic switching on of the field, so for simplicity we set $\epsilon=0$. This is possible for a reasonable laser detuning. Further, we adopt a global lifetime $\gamma_{n m}=\gamma$, thus associating this damping parameter with the external frequency $\omega$, rather than the transition specific frequency $\omega_{n 0}$. With the interpretation of the damping parameter as discussed above, a global parameter would imply that the average lifetime of all excited states of the molecular system is identical. While this is strictly not true, we will show that it results not only in more tractable expressions, but it also carries with it potentially large computational savings (especially for the approach described in Section 3.2.3).

By comparing the above expression to Eq. 3.7, the linear response function is found to be equal to

$$
\left\langle\left\langle\hat{\Omega} ; \hat{V}_{\beta}^{\omega}\right\rangle\right\rangle_{\omega}=-\frac{1}{\hbar} \sum_{n}\left[\frac{\langle 0|\hat{\Omega}| n\rangle\left\langle n\left|\hat{V}_{\beta}^{\omega}\right| 0\right\rangle}{\omega_{n 0}-\omega-i \gamma}+\frac{\left\langle 0\left|\hat{V}_{\beta}^{\omega}\right| n\right\rangle\langle n|\hat{\Omega}| 0\rangle}{\omega_{n 0}+\omega+i \gamma}\right] .
$$

Returning to our discussion of the divergent nature of (standard) response theory lacking phenomenological damping, or relaxation, described here, it is possible to arrive to an equivalent expression for the linear response function. This expression differs only in that the damping term $\gamma=0$, and the response function as a whole is thus real. As can be seen in the denominators, we thus have singularities at resonance. In other words, by letting the frequency $\omega \rightarrow \omega+i \gamma$, we obtain a complex response function which allows for calculations including resonance frequencies.

With this damped linear response function, the polarizability is given by the case where an electric dipole moment operator is the operator corresponding to the perturbing field and the observable. With this Eq. 3.20 corresponds to the complex polarization propagator (CPP) and the complex polarizability is given as

$$
\alpha_{\alpha \beta}(-\omega ; \omega)=\alpha_{\alpha \beta}^{\mathrm{I}}(-\omega ; \omega)+i \alpha_{\alpha \beta}^{\mathrm{R}}(-\omega ; \omega)=-\left\langle\left\langle\hat{\mu}_{\alpha} ; \hat{\mu}_{\beta}\right\rangle\right\rangle_{\omega} .
$$

In Fig. 3.1 the behaviour of the real and imaginary part of this complex polarizability, as obtained for a single excited state in Eq. 3.20, is illustrated at and in the vicinity of resonance.

The absorption cross-section, and thus spectrum of a system, can be obtained by the imaginary part of the polarizability

$$
\sigma(\omega)=\frac{4 \pi \omega}{3 c} \sum_{\beta=x, y, z} \alpha_{\beta \beta}^{\mathrm{I}}(-\omega ; \omega),
$$

for all Cartesian component $\beta$ of the electric dipole moment operator and $c$ is the speed of light.

The imaginary part of the polarizability is seen to be equal to

$$
\alpha_{\alpha \beta}^{\mathrm{I}}=\frac{\gamma}{\hbar} \sum_{n}\left[\frac{\left\langle 0\left|\hat{\mu}_{\alpha}\right| n\right\rangle\left\langle n\left|\hat{\mu}_{\beta}\right| 0\right\rangle}{\left(\omega_{0 n}-\omega\right)^{2}+\gamma^{2}}-\frac{\left\langle 0\left|\hat{\mu}_{\beta}\right| n\right\rangle\left\langle n\left|\hat{\mu}_{\alpha}\right| 0\right\rangle}{\left(\omega_{0 n}+\omega\right)^{2}+\gamma^{2}}\right],
$$




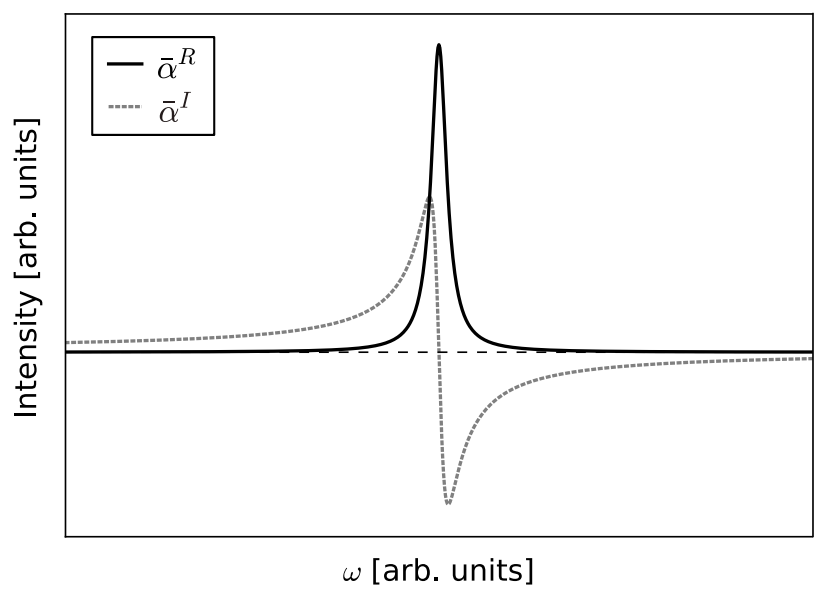

Figure 3.1. The real and imaginary part of the complex polarizability at and in the vicinity of resonance.

where, at resonance, the leading term is

$$
f\left(\omega ; \omega_{n 0}, \gamma\right)=\frac{A}{\pi} \frac{\gamma}{\left(\omega_{0 n}-\omega\right)^{2}+\gamma^{2}}, \quad \text { with } \quad A=\pi \frac{\left\langle 0\left|\hat{\mu}_{\alpha}\right| n\right\rangle\left\langle n\left|\hat{\mu}_{\beta}\right| 0\right\rangle}{\hbar}
$$

corresponding to a Lorentzian function with amplitude $A /(\pi \gamma)$ and a half-width at half-maximum of $\gamma$.

Thus, the inclusion of phenomenological damping by (the inverse of) a finite lifetime $\gamma$ yields a Lorentzian broadening. For any measurement, the excitation peaks are broadened by a number of processes, including already considered spontaneous and stimulated emission, molecular vibrations and the finite resolution of the experimental equipment (resulting in an asymmetric and Gaussian broadening, respectively [4]), as well as through Doppler shift due to the relative movements and thermal vibrations of the molecules [33]. As a result, the theoretical Lorentzian broadening will not correspond exactly to the broadening of experimental spectra - the other effects will in most cases dominate over lifetime broadening, especially for significant temperatures. Nonetheless, the spectral features will be close to that of experiment and additional effects can be addressed if desired, e.g. by performing response calculations on snapshots from dynamics.

\subsubsection{Quasi-energy formalism}

As was explained in the beginning of this chapter, identifying molecular properties with energy derivatives is a valid approach for static perturbations, but this approach is not possible for time-dependent perturbations owing to the ill-defined energies of such interactions. There exists, however, a similar property we can expand in orders of the perturbation instead, namely the quasi-energy. 
First, let us reformulate the wave function as a product of two time-dependent functions

$$
|\psi(t)\rangle=e^{-i \phi(t)}|\bar{\psi}(t)\rangle
$$

The choice of these functions are made unique by requiring $\phi(t)$ to be a real function and the phase of the projection of $\bar{\psi}(t)$ onto ground state $\psi_{0}$ zero. This can be compared to the time-evolution of the unperturbed (static) system, which is given by variable separation in the Schrödinger equation 2.1 as

$$
|\psi(t)\rangle=e^{-i E_{0} t / \hbar}|0\rangle
$$

with $|0\rangle$ as the ground state wave function. Inserting Eq. 3.25 in the Schrödinger equation yields

$$
\left(\hat{H}-i \hbar \frac{\partial}{\partial t}\right)|\bar{\psi}(t)\rangle=Q(t)|\bar{\psi}(t)\rangle=\hbar \dot{\phi}(t)|\bar{\psi}(t)\rangle
$$

where $Q(t)$ is the time-dependent quasi-energy. This property can in principle be calculated by the projection

$$
Q(t)=\left\langle\bar{\psi}(t)\left|\left(\hat{H}-i \hbar \frac{\partial}{\partial t}\right)\right| \bar{\psi}(t)\right\rangle
$$

and thus refers to to expectation value of the difference between the time and Hamiltonian operators.

The requirements of Eq. 3.25 and the definition of $Q(t)$ in Eq. 3.27 results in the following expression in the absence of perturbing fields:

$$
\begin{aligned}
& |\bar{\psi}(t)\rangle=\left|\psi_{0}\right\rangle, \\
& \phi(t)=E_{0} t / \hbar, \\
& Q(t)=E_{0} .
\end{aligned}
$$

For the phase-isolated wave function, phase function and quasi-energy, respectively. We also define the time-averaged quasi-energy as

$$
Q_{T}=\frac{1}{T} \int_{t}^{t+T} Q\left(t^{\prime}\right) d t^{\prime}
$$

where $T$ is the period time.

If we consider times when only the fundamental frequencies remains to be accounted for, the time-averaged quasi-energy will have no dependencies on time. In a manner analogously to Eqs. 3.2 and 3.7, the time-averaged quasi-energy is 
expanded in orders of the external perturbation as

$$
\begin{aligned}
Q_{T}\left(F^{\omega}\right) & =E_{0} \\
& +\left.\sum_{\omega_{1}} \frac{d Q_{T}}{d F_{\alpha}^{\omega_{1}}}\right|_{F^{\omega}=0} F_{\alpha}^{\omega_{1}} \\
& +\left.\frac{1}{2 !} \sum_{\omega_{1} \omega_{2}} \frac{d^{2} Q_{T}}{d F_{\alpha}^{\omega_{1}} d F_{\beta}^{\omega_{2}}}\right|_{F^{\omega}=0} F_{\alpha}^{\omega_{1}} F_{\beta}^{\omega_{2}} \\
& +\left.\frac{1}{3 !} \sum_{\omega_{1} \omega_{2} \omega_{3}} \frac{d^{3} Q_{T}}{d F_{\alpha}^{\omega_{1}} d F_{\beta}^{\omega_{2}} d F_{\gamma}^{\omega_{3}}}\right|_{F^{\omega}=0} F_{\alpha}^{\omega_{1}} F_{\beta}^{\omega_{2}} F_{\gamma}^{\omega_{3}} \\
& +\ldots
\end{aligned}
$$

From these derivatives we can thus identify the different molecular properties. As an example, the first-order correction is calculated as

$$
\frac{d Q_{T}}{d F_{\alpha}^{\omega}}=\frac{1}{T} \int_{t}^{t+T}\left\langle\bar{\psi}\left(t^{\prime}\right)\left|\frac{\partial \hat{H}}{\partial F_{\alpha}^{\omega}}\right| \bar{\psi}\left(t^{\prime}\right)\right\rangle d t^{\prime}=\frac{1}{T} \int_{t}^{t+T}\left\langle\bar{\psi}\left(t^{\prime}\right)\left|\hat{V}_{\alpha}^{\omega}\right| \bar{\psi}\left(t^{\prime}\right)\right\rangle e^{-i \omega t^{\prime}} e^{\epsilon t^{\prime}} d t^{\prime},
$$

which is the Hellmann-Feynman theorem for time-independent quasi-energy.

\subsection{Damped coupled cluster linear response the- ory}

With the exact state linear response function obtained, we will now derive the (real) coupled cluster linear response function. This derivation starts with the quasi-energy concept and utilize Lagrangian multipliers to ensure that the wave function is normalized. Once the real linear response function is derived, the complex counterpart is be found by the substitution $\omega \rightarrow \omega+i \gamma$, and we will then proceed to two computational schemes by which the response function can be calculated, the asymmetric Lanczos-chain method and iteratively constructing a reduced subspace for direct inversion. The chapter will then end with a discussion concerning the advantages and disadvantages of the two approaches.

\subsubsection{Damped coupled cluster linear response function}

As previously discussed, explicit response expressions for exact states can be formulated using both the Ehrenfest and quasi-energy formulations. However, for non-variational electronic structure methods such as coupled cluster, the Ehrenfest formalism can no longer be used [31]. We will thus discuss the derivation of linear response equations in coupled cluster using quasi-energy [31,34], for which Eq. 3.27 becomes

$$
\left(\hat{H}-i \hbar \frac{\partial}{\partial t}\right) e^{\hat{T}(t)}|0\rangle=Q(t) e^{\hat{T}(t)}|0\rangle
$$


With the time-derivative of the phase-isolated coupled cluster state being orthogonal to the reference HF state, the quasi-energy can be isolated as

$$
Q(t)=\left\langle 0\left|\left(\hat{H}-i \hbar \frac{\partial}{\partial t}\right) e^{\hat{T}(t)}\right| 0\right\rangle=\left\langle 0\left|\hat{H} e^{\hat{T}(t)}\right| 0\right\rangle .
$$

This expression can be regarded as the quasi-energy analogue of the coupled cluster energy equation 2.13. Likewise, projecting Eq. 3.35 onto the excitation manifold analogous to Eq. 2.14 yields the time-dependent amplitudes as

$$
0=\left\langle n\left|e^{-\hat{T}(t)}\left(\hat{H}-i \hbar \frac{\partial}{\partial t}\right) e^{\hat{T}(t)}\right| 0\right\rangle .
$$

It should be noted that the quasi-energy isolated above is no longer guaranteed to be real, as this projection is not fully analogous with the expectation value expressed by Eq. 3.28. Care must be taken to ensure that the response equations derived from this property are well defined.

The coupled cluster response functions are now sought using the quasi-energy in Eq. 3.36, with the constraints imposed by Eq. 3.37. The constraints can be incorporated by means of a variational Lagrangian technique, where equations of motion must fulfill the variational conditions

$$
\delta L_{T}=0,
$$

for the time-averaged Lagrangian. This time-averaged Lagrangian can be formed from the coupled cluster Lagrangian in the same manner as the time-averaged quasi-energy of Eq. 3.32, with a time-dependent Lagrangian equal to

$$
L(t)=Q(t)+\sum_{n} \lambda_{n}(t)\left\langle n\left|e^{-\hat{T}(t)}\left(\hat{H}-i \hbar \frac{\partial}{\partial t}\right) e^{\hat{T}(t)}\right| 0\right\rangle .
$$

The time-dependent Lagrangian multipliers $\lambda_{n}$ are here associated with the constraints given by a specific excited determinant $n$.

We now seek the derivatives of $L_{T}$ in order to determine molecular property, as can be compared to the expansion time-averaged quasi-energy in Eq. 3.33, and we first consider the zeroth-order response function

$$
\left\langle\hat{V}_{\alpha}^{\omega_{1}}\right\rangle=\left.\frac{d L_{T}}{d F_{\alpha}^{\omega_{1}}}\right|_{F^{\omega}=0} .
$$

In order to evaluate this field derivative, the derivative of the time-averaged Lagrangian with respect to the Lagrangian multipliers and the amplitudes are first required. With the variational condition 3.38 the derivatives with respect to the multipliers are equal to zero, i.e.

$$
\left.\frac{\partial L_{T}}{\partial \lambda_{n}^{(0)}}\right|_{F^{\omega}=0}=\left\langle n\left|e^{-\hat{T}^{(0)}} \hat{H}_{0} e^{\hat{T}^{(0)}}\right| 0\right\rangle=0,
$$

determining the amplitudes of the $\mathrm{CC}$ reference state in a manner similar to Eq. 3.37. Superscripts designate the $N$ :th order correction with respect to the perturbation. 
For the amplitude derivatives the variational condition instead gives

$$
\left.\frac{\partial L_{T}}{\partial t_{n}^{(0)}}\right|_{F^{\omega}=0}=\left\langle 0\left|\hat{H}_{0} \hat{\tau}_{n}^{\dagger}\right| \mathrm{CC}\right\rangle+\sum_{n} \lambda_{k}^{(0)}\left\langle k\left|e^{-\hat{T}^{(0)}}\left[\hat{H}_{0}, \hat{\tau}_{n}^{\dagger}\right] e^{\hat{T}}\right| 0\right\rangle=0,
$$

where $\hat{\tau}_{n}^{\dagger}$ is the excitation operator corresponding to amplitude $t_{n}$, which is given as

$$
t_{n}(0)=t_{n}^{(0)}+\sum_{\omega_{1}} t_{n}^{(1)} e^{-i \omega_{1} t}+\ldots
$$

In order to obtain the zeroth-order multipliers, we see that we can rearrange and then rewrite Eq. 3.42 in matrix form, such that the multipliers in vector form is given as

$$
\lambda^{(0)}=-\kappa^{[1]}\left[\mathbf{A}^{[2]}\right]^{-1},
$$

with

$$
\kappa_{n}^{[1]}=\left\langle 0\left|\hat{H}_{0} \hat{\tau}_{n}^{\dagger} e^{\hat{T}}\right| 0\right\rangle \quad \text { and } \quad A_{k n}^{[2]}=\left\langle k\left|e^{-\hat{T}^{(0)}}\left[\hat{H}_{0}, \hat{\tau}_{n}^{\dagger}\right] e^{\hat{T}}\right| 0\right\rangle,
$$

the matrix $\mathbf{A}^{[2]}$ here is the non-symmetric coupled cluster Jacobian.

The zeroth-order response functions are now calculated via Eq. 3.40, for which the field derivatives are found to be

$$
\begin{aligned}
\left.\frac{d L_{T}}{d F_{\alpha}^{\omega_{1}}}\right|_{F^{\omega}=0} & =\left.\frac{\partial L_{T}}{\partial F_{\alpha}^{\omega_{1}}}\right|_{F^{\omega}=0}+\left.\sum_{n}\left[\frac{\partial L_{T}}{\partial t_{n}^{(1)}} \frac{\partial t_{n}^{(1)}}{\partial F_{\alpha}^{\omega_{1}}}+\frac{\partial L_{T}}{\partial \lambda_{n}^{(1)}} \frac{\partial \lambda_{n}^{(1)}}{\partial F_{\alpha}^{\omega_{1}}}\right]\right|_{F^{\omega}=0} \\
= & \left.\frac{\partial L_{T}}{\partial F_{\alpha}^{\omega_{1}}}\right|_{F^{\omega}=0}=\left[\left\langle 0\left|\hat{V}_{\alpha}^{\omega_{1}} e^{\hat{T}^{(0)}}\right| 0\right\rangle+\sum_{n} \lambda_{n}^{(0)}\left\langle n\left|e^{-\hat{T}^{(0)}} \hat{V}_{\alpha}^{\omega_{1}} e^{\hat{T}^{(0)}}\right| 0\right\rangle\right] \delta_{\omega_{1}}
\end{aligned}
$$

where the evaluation is made at zero field strength and the variational condition ensures that derivatives with respect to amplitudes and multipliers in the first row vanish.

With the zeroth-order response function derived, and the zeroth-order multipliers given by Eq. 3.44, we continue with the linear response function, obtained from the field derivative

$$
\left\langle\left\langle\hat{V}_{\alpha}^{\omega_{1}} ; \hat{V}_{\beta}^{\omega_{2}}\right\rangle\right\rangle=\left.\frac{d^{2} L_{T}}{d F_{\alpha}^{\omega_{1}} d F_{\beta}^{\omega_{2}}}\right|_{F^{\omega}=0} .
$$

These functions are determined by further derivations of Eq. 3.46 with respect to the external field and to ensure that the expression is symmetric a permutation operator $P_{\alpha, \beta}$ is introduced (acting by permuting the field amplitudes $F_{\alpha}^{\omega_{1}}$ and $\left.F_{\beta}^{\omega_{2}}\right)$. By cancellation of terms and implicit summation over indices, the linear response function is found to be

$$
\begin{aligned}
\left\langle\left\langle\hat{V}_{\alpha}^{\omega_{1}} ; \hat{V}_{\beta}^{\omega_{2}}\right\rangle\right\rangle & =\left.\frac{d^{2} L_{T}}{d F_{\alpha}^{\omega_{1}} d F_{\beta}^{\omega_{2}}}\right|_{F^{\omega}=0} \\
& =\sum P_{\alpha, \beta}\left[\left.\frac{\partial^{2} L_{T}}{\partial F_{\alpha}^{\omega_{1}} \partial t_{n}^{(1)}} \frac{\partial t_{n}^{(1)}}{\partial F_{\beta}^{\omega_{2}}}\right|_{F^{\omega}=0}+\left.\frac{1}{2} \frac{\partial t_{m}^{(1)}}{\partial F_{\alpha}^{\omega_{1}}} \frac{\partial^{2} L_{T}}{\partial t_{m}^{(1)} \partial t_{n}^{(1)}} \frac{\partial t_{n}^{(1)}}{\partial F_{\beta}^{\omega_{2}}}\right|_{F^{\omega}=0}\right]
\end{aligned}
$$


The time-averaged Lagrangian derivatives can be expressed as

$$
\begin{gathered}
\frac{\partial^{2} L_{T}}{\partial F_{\alpha}^{\omega_{1}} \partial t_{n}^{(1)}\left(\omega_{2}\right)}=\left[\left\langle 0\left|\hat{V}_{\alpha}^{\omega_{1}} \hat{\tau}_{n}^{\dagger} e^{\hat{T}}\right| 0\right\rangle+\sum_{k} \lambda_{k}^{(0)}\left\langle k\left|e^{-\hat{T}^{(0)}}\left[\hat{V}_{\alpha}^{\omega_{1}}, \hat{\tau}_{n}^{\dagger}\right] e^{\hat{T}}\right| 0\right\rangle\right] \delta_{\omega_{1}+\omega_{2}} \\
\frac{\partial^{2} L_{T}}{\partial t_{m}^{(1)}\left(\omega_{1}\right) \partial t_{n}^{(1)}\left(\omega_{2}\right)}=\left[\left\langle 0\left|\hat{H}_{0} \hat{\tau}^{\dagger} \hat{\tau}_{n}^{\dagger} e^{\hat{T}}\right| 0\right\rangle+\sum_{k} \lambda_{k}^{(0)}\left\langle k\left|e^{-\hat{T}^{(0)}}\left[\left[\hat{H}_{0}, \hat{\tau}_{m}^{\dagger}\right], \hat{\tau}_{n}^{\dagger}\right] e^{\hat{T}}\right| 0\right\rangle\right] \delta_{\omega_{1}+\omega_{2}}
\end{gathered}
$$

In order to evaluate the field derivative of the first-order amplitudes at zero field strength the variational condition 3.38 is used repeatedly, such

$$
\frac{d}{d F_{\alpha}^{\omega_{1}}}\left[\frac{\partial L_{T}}{\partial \lambda_{m}^{(1)}\left(\omega_{2}\right)}\right]=\frac{\partial^{2} L_{T}}{\partial F_{\alpha}^{\omega_{1}} \partial \lambda_{m}^{(1)}}+\frac{\partial^{2} L_{T}}{\partial \lambda_{m}^{(1)} \partial t_{n}^{(1)}} \frac{\partial t_{n}^{(1)}}{\partial F_{\alpha}^{\omega_{1}}},
$$

from which the amplitudes are calculated as the matrix-vector product

$$
\left.\frac{\partial t^{(1)}\left(\omega_{1}\right)}{\partial F_{\alpha}^{\omega_{1}}}\right|_{F^{\omega}=0}=-\left.\left[\left.\frac{\partial^{2} L_{T}}{\partial \lambda^{(1)} \partial t^{(1)}}\right|_{F^{\omega}=0}\right]^{-1} \frac{\partial^{2} L_{T}}{\partial F_{\alpha}^{\omega_{1}} \partial \lambda^{(1)}}\right|_{F^{\omega}=0}=0 .
$$

The elements of the right-hand-side matrix and vector are expressed as

$$
\begin{aligned}
& \left.\frac{\partial^{2} L_{T}}{\partial \lambda^{(1)}\left(\omega_{1}\right) \partial t^{(1)}\left(\omega_{1}\right)}\right|_{F^{\omega}=0}=\left[A_{m n}^{[2]}-\hbar \omega_{2} \delta_{m n}\right] \delta_{\omega_{1}+\omega_{2}}, \\
& \left.\frac{\partial^{2} L_{T}}{\partial F_{\alpha}^{\omega_{1}} \partial \lambda_{m}^{(1)}\left(\omega_{1}\right)}\right|_{F^{\omega}=0}=\left\langle m\left|e^{-\hat{T}^{(0)}} \hat{V}_{\alpha}^{\omega_{1}} e^{\hat{T}}\right| 0\right\rangle \delta_{\omega_{1}+\omega_{2}}=\xi,
\end{aligned}
$$

where the last identification is tailored for the following, simplified expression of Eq. 3.52

$$
\left(\mathbf{A}^{[2]}-\hbar \omega_{2} \mathbf{1}\right) t(\omega)=-\xi .
$$

This is the linear response equation that needs to be solved in a efficient manner, with the Jacobian being of such size that the direct inversion of the matrix at the left size in unfeasible. Written in this form, we also notice that the singularities of the response function occurs when the matrix to the left is singular, meaning that the excitation energies can be determined by diagonalizing this Jacobian.

In order to avoid these singularities and get a resonance-convergent method, we incorporate relaxation in the response function in the manner discussed concerning Eq. 3.12, by letting $\omega \rightarrow \omega+i \gamma$. This results in the complex linear response equation

$$
\left(\mathbf{A}^{[2]}-\hbar(\omega+i \gamma) \mathbf{1}\right) t(\omega+i \gamma)=-\xi
$$

As the amplitude derivative vector $t$ cannot be found by direct inversion due to the size of the coupled cluster Jacobian, we will now discuss two other approaches by which the damped CC linear response function can be calculated. 


\subsubsection{The asymmetric Lanczos-chain method}

An established, iterative method of transforming a large matrix to a smaller, tridiagonal form is the Lanczos-based algorithm (or the Lanczos-chain method), described in Ref. [35] and implemented in a symmetrical setting in Ref. [36]. With this, the entire spectral region is iteratively obtained, starting with the extremal, most intense transitions. This approach for the solution of the damped coupled cluster linear response function have been implemented and the performance evaluated in Papers I-III. Some aspects of this approach will now be discussed, largely following Paper II, but with some differences in notation.

Consider an asymmetric Jacobian matrix $\mathbf{A}^{[2]}$ of dimension $n$. We seek a transformation of this matrix such that

$$
\mathbf{T}^{(m)}=\mathbf{P}^{(m)^{T}} \mathbf{A}^{[2]} \mathbf{Q}^{(m)},
$$

where $\mathbf{T}^{(m)}$ is of dimension $m(\leq n)$ and has an asymmetric tri-diagonal form such that

$$
\mathbf{T}^{(m)}=\left(\begin{array}{ccccc}
\alpha_{1} & \gamma_{1} & 0 & & 0 \\
\beta_{1} & \alpha_{2} & & \ddots & \\
0 & & \ddots & & 0 \\
& \ddots & & \alpha_{m-1} & \gamma_{m} \\
0 & & 0 & \beta_{m} & \alpha_{m}
\end{array}\right)
$$

The transformation matrices $\mathbf{P}^{(m)}$ and $\mathbf{Q}^{(m)}$ are of dimension $n \times m$ and biorthonormal

$$
\mathbf{P}^{(m), T}=\mathbf{Q}^{(m),-1} \quad \Rightarrow \quad \mathbf{P}^{(m), T} \mathbf{Q}^{(m)}=\mathbf{1}
$$

If the transformation is made in full dimension, i.e. $m=n$, the exact tridiagonal representation is obtained, with properties such that

$$
\mathbf{A}^{[2]} \mathbf{Q}^{(n)}=\mathbf{Q}^{(n)} \mathbf{T}^{(n)} \quad \text { and } \quad \mathbf{A}^{[2], T} \mathbf{P}^{(n)}=\mathbf{P}^{(n)} \mathbf{T}^{(n), T}
$$

By labeling the columns in $\mathbf{Q}^{(n)}$ as $\bar{q}_{1}, \bar{q}_{2}, \ldots, \bar{q}_{n}$, and analogous for $\mathbf{P}^{(n)}$, the matrices and elements in $\mathbf{T}^{(n)}$ can be found to fulfill the relations

$$
\begin{aligned}
\mathbf{A}^{[2]} \bar{q}_{i} & =\gamma_{i-1} \bar{q}_{i-1}+\alpha_{i} \bar{q}_{i}+\beta_{i} \bar{q}_{i+1}, \\
\mathbf{A}^{[2], T} \bar{p}_{i} & =\beta_{i-1} \bar{p}_{i-1}+\alpha_{i} \bar{p}_{i}+\gamma_{i} \bar{p}_{i+1},
\end{aligned}
$$

for all $i=1, \ldots, n-1$, with the requirement that $\gamma_{0} \bar{q}_{0}=\beta_{0} \bar{p}_{0}=0$. It is thus possible to generate new vectors $\bar{q}_{i+1}$ and $\bar{p}_{i+1}$ recursively, by using the information obtained by previous iterations such that

$$
\begin{aligned}
& \beta_{i} \bar{q}_{i+1}=r_{i}=\left(\mathbf{A}^{[2]}-\alpha_{i} \mathbf{1}\right) \bar{q}_{i}-\gamma_{i} \bar{q}_{i+1}, \\
& \gamma_{i} \bar{p}_{i+1}=s_{i}=\left(\mathbf{A}^{[2], T}-\alpha_{i} \mathbf{1}\right) \bar{p}_{i}-\beta_{i} \bar{p}_{i+1} .
\end{aligned}
$$


The parameters $\alpha_{i}, \beta_{i}$, and $\gamma_{i}$ can be obtained from the bi-orthonormality condition 3.59, where $\alpha_{i}$ has a definite value and the remaining two parameters are related

$$
\alpha_{i}=\bar{p}_{i}^{T} \mathbf{A}^{[2]} \bar{q}_{i} \quad \text { and } \quad \bar{p}_{i}^{T} \bar{q}_{i}=\left(\frac{s_{i}}{\gamma_{i}}\right)\left(\frac{r_{i}}{\beta_{i}}\right)=1 .
$$

For a calculation it is customary to enforce the latter condition (with $\delta_{i j}$ to the right) between all Lanczos vectors $\bar{q}_{i}$ and $\bar{p}_{j}$, as numerical noise would otherwise make the algorithm unstable for large dimensions.

If the calculation of the tri-diagonal matrix is halted at a dimension $m<n$, also designated at a chain length $m$, an error is introduced and $\mathbf{T}^{(m)}$ is only an approximation of the tri-diagonal matrix. In this case the first relation of Eq. 3.60 is now found to be

$$
\mathbf{A}^{[2]} \mathbf{Q}^{(m)}=\mathbf{Q}^{(m)} \mathbf{T}^{(m)}+\beta_{m} \bar{q}_{m+1} \bar{e}_{m}^{(m), T},
$$

where the last term is the truncation error, with $\bar{e}_{m}^{(m)}$ being a unit vector with 1 in element $m$.

Neglecting the truncation error, an approximate Jacobian $\mathbf{A}^{(m)}$ is constructed as

$$
\mathbf{A}^{(m)} \mathbf{Q}^{(m)}=\mathbf{Q}^{(m)} \mathbf{T}^{(m)},
$$

ant the tri-diagonal matrix is diagonalized with left and right eigenvectors

$$
\begin{aligned}
& \mathbf{T}^{(m)} \mathbf{R}^{(m)}=\mathbf{R}^{(m)} \boldsymbol{\Omega}^{(m)}, \\
& \mathbf{L}^{(m)} \mathbf{T}^{(m)}=\boldsymbol{\Omega}^{(m)} \mathbf{L}^{(m)},
\end{aligned}
$$

where $\boldsymbol{\Omega}^{(m)}$ is a diagonal matrix with the $m$ eigenvalues of $\mathbf{T}^{(m)}$. Using these eigenvectors the approximate eigenvectors of $\mathbf{A}^{[2]}$ are given as

$$
\begin{aligned}
& \mathbf{X}_{L}^{(m)}=\mathbf{L}^{(m)} \mathbf{P}^{(m), T}, \\
& \mathbf{X}_{R}^{(m)}=\mathbf{Q}^{(m)} \mathbf{R}^{(m)} .
\end{aligned}
$$

This eigenvectors corresponds to the excitation vectors, and can thus be used to study the nature of the excitation, use as the basis for a perturbative correction of the excitation energies etc.

For details on how the Lanczos algorithm is implemented in coupled cluster damped linear response theory, see Paper II and Ref. [5]. Included there are specifications on the choice of $\bar{q}_{i}$ and $\bar{p}_{i}$, including initial vectors, and the procedure of how Eq. 3.48 can be rewritten to the following form

$$
\begin{aligned}
\left\langle\left\langle\hat{\Omega} ; \hat{V}_{\beta}^{\omega}\right\rangle\right\rangle_{\omega}= & N_{\eta} N_{\xi} \sum_{j}\left[\frac{L_{j 1}^{(m)} R_{1 j}^{(m)}}{\omega-\omega_{j}+i \gamma}-\frac{L_{j 1}^{(m)} R_{1 j}^{(m)}}{\omega+\omega_{j}+i \gamma}\right] \\
& +N_{\xi}^{2} \sum_{j k} F_{k j} \frac{L_{j 1}^{(m)} L_{k 1}^{(m)}}{\left(\omega-\omega_{j}-i \gamma\right)\left(\omega-\omega_{k}+i \gamma\right)}
\end{aligned}
$$


where $N_{\eta}$ and $N_{\xi}$ are normalization factors, $\mathbf{L}^{(m)}$ and $\mathbf{R}^{(m)}$ the Lanczos eigenvectors, and $\mathbf{F}$ can be found in Paper II. Also note that atomic units are used.

From this expression, we can thus calculate directly any spectrum of interest, with broadening included. In addition to this, it is also possible to calculate the approximate coupled cluster eigenvectors in a manner analogously to Eqs. 3.70 and 3.71, and from these we can get the explicit excited states with information concerning transition energies, oscillator strengths and the character of the excitations.

The main difficulty with the Lanczos algorithm is to ensure that the spectral region of interest is sufficiently well converged. If an insufficient chain length is chosen, it is possible that several less intense features are combined into one, incorrect, spectral feature, and care must be taken to ensure that this is not the case.

As an illustration of the convergence properties of the algorithm, we have calculated the spectrum of neon at the CCSD/t-aug-cc-pVTZ level of theory using different Lanczos chain lengths $j$, with the results shown in Fig. 3.2. The spectral features converge from the lowest and highest eigenvalues, with the features at the first $100 \mathrm{eV}$ being very similar for $j=200$ and $j=800$. This is a property of the Lanczos algorithm and for the case of X-ray absorption spectroscopy it means that we need a large chain length to converge the core excited states (in this case at around $870 \mathrm{eV}$ ), as the valence states need to be converged first. In this context, we also note the difference in intensity between the low-energy states and those at around $870 \mathrm{eV}$ (which are barely visible), this is due to the larger overlap of valence and excited state orbitals than of core and excited state orbitals. As the chain length is increased, the maximum of the peaks are observed to decrease, as the previously unconverged transitions are no longer summed into one larger one (of an intermediate energy).

As the Lanczos algorithm is a method by which the global spectrum is converged iteratively, it is possible to calculate the global integrated absorption crosssection $\mathrm{I}_{\mathrm{S}}$. For a variational method this value should be equal to the total number of electrons, as given by the Thomas-Reiche-Kuhn sum rule. For the results illustrated in Fig. 3.2, the $\mathrm{I}_{\mathrm{S}}$ values are reported to be equal to 10.19, differing by most 0.0002 for the different chain lengths. This is in line with previous observationsthe algorithm combine several low-intensity features into a stronger feature, conserving the total transition moment or integrated absorption cross-section. As an extreme example, a Lanczos CPP-CCSD calculation was carried out using a chain length $j=1$, for which all the aborption cross-section is combined into one feature, resulting in a $I_{S}$ value of 10.19. It is thus clear that the Lanczos CPP approach will give the converged $\mathrm{I}_{\mathrm{S}}$ value using any chain length, and discrepancies in the values as compared to the Thomas-Reich-Kuhn sum rule is thus a result of the electronic structure and basis set used. 


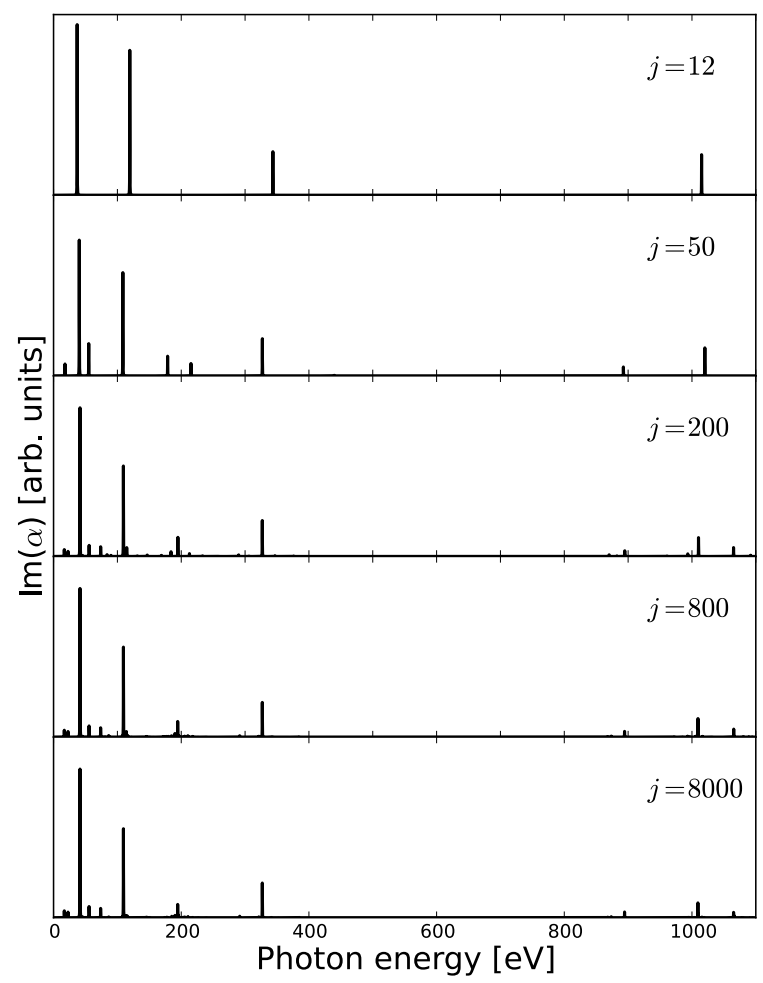

Figure 3.2. The global spectrum of neon, as calculated using CCSD damped linear response theory with different chain lengths $j$ in the Lanczos algorithm. The spectral region is set as $0-1100 \mathrm{eV}$ and the basis set is chosen as t-aug-cc-pVTZ.

\subsubsection{Direct solver in a reduced subspace}

As an alternative to the Lanczos-chain-driven algorithm described above, a direct solver scheme of the complex response equation, or CPP, in a reduced subspace is being developed [37]. In this scheme, an iterative subspace algorithm is used to construct a subspace in which arbitrary frequencies can be addressed. This approach have been established earlier for other spectroscopies and electronic structure methods, see e.g. Ref. [38] for an implementation in four-component relativistic, noncollinear KS-DFT, or Ref. [39] for a discussion of efficient implementations in HF and KS-DFT.

The scheme addresses the solution of the linear response equation 3.56, for which we explicitly rewrite the solution vector and right-hand-side in the complex vectors, respectively

$$
t=t^{R}+i t^{I}, \quad \xi=\xi^{R}+i \xi^{I} .
$$

Note that $\xi^{I}=0$ for real operators, as is the case for the determination of complex 
polarizability. This yields the response equation

$$
\left(\mathbf{A}^{[2]}-\hbar(\omega+i \gamma) \mathbf{1}\right)\left(t^{R}+i t^{I}\right)=-\left(\xi^{R}+i \xi^{I}\right) .
$$

This equation can be written in a real matrix form as

$$
\left(\begin{array}{cc}
\left(\mathbf{A}^{[2]}-\hbar \omega \mathbf{1}\right) & \hbar \gamma \mathbf{1} \\
\hbar \gamma \mathbf{1} & -\left(\mathbf{A}^{[2]}-\hbar \omega \mathbf{1}\right)
\end{array}\right)\left(\begin{array}{c}
t^{R} \\
t^{I}
\end{array}\right)=\left(\begin{array}{c}
-\xi^{R} \\
\xi^{I}
\end{array}\right) .
$$

We seek to solve Eq. 3.75 using an iterative subspace algorithm, for which, at $k$ :th iteration, a subspace of trial vectors has been constructed such that

$$
\begin{aligned}
b^{n} & =\left\{b_{1}^{R}, b_{1}^{I}, \ldots, b_{n / 2}^{R}, b_{n / 2}^{I}\right\} \\
& =\left\{b_{1}, b_{2}, \ldots, b_{n-1}, b_{n}\right\}
\end{aligned}
$$

where the subspace for the real and imaginary solution vectors are mixed, as this may improve convergence. In practice, it is most likely not the case that we have as many trial vectors that originate from real and imaginary trial vectors, due to orthonormalization procedures, and we also note that such operations ensures that the size of the subspace $n \leq k$.

For each trial vector, a corresponding linear transformed vector is formed, yielding the set

$$
\sigma^{n}=\left\{\mathbf{A}^{[2]} b_{1}, \mathbf{A}^{[2]} b_{2}, \ldots, \mathbf{A}^{[2]} b_{n-1}, \mathbf{A}^{[2]} b_{n}\right\} .
$$

With this at hand, the reduced real matrix equation is constructed as

$$
\left(\begin{array}{cc}
\left(\mathbf{A}_{\text {red }}^{[2]}-\hbar \omega \mathbf{1}_{\text {red }}\right) & \hbar \gamma \mathbf{1}_{\text {red }} \\
\hbar \gamma \mathbf{1}_{\text {red }} & -\left(\mathbf{A}_{\text {red }}^{[2]}-\hbar \omega \mathbf{1}_{\text {red }}\right)
\end{array}\right)\left(\begin{array}{c}
t_{\text {red }}^{R} \\
t_{\text {red }}^{I}
\end{array}\right)=\left(\begin{array}{c}
-\xi_{\text {red }}^{R} \\
\xi_{\text {red }}^{I}
\end{array}\right),
$$

with

$$
\left[\xi_{\text {red }}^{R}\right]_{i}=b_{i}^{T} \xi^{R}, \quad\left[\xi_{\text {red }}^{I}\right]_{i}=b_{i}^{T} \xi^{I}, \quad\left[\mathbf{A}_{\text {red }}^{[2]}\right]_{i j}=b_{i}^{T} \mathbf{A}^{[2]} b_{j}=b_{i}^{T} \sigma_{j} .
$$

The reduced matrix equation is solved by inversion, and by this the optimal solution vectors for iteration $k$ are calculated as

$$
t_{\mathrm{opt}}^{R}=\sum_{i=1}^{n}\left[t_{\mathrm{red}}^{R}\right]_{i} b_{i} \quad \text { and } \quad t_{\mathrm{opt}}^{I}=\sum_{i=1}^{n}\left[t_{\mathrm{red}}^{I}\right]_{i} b_{i} .
$$

Using these solution vectors the residual is calculated as

$$
\left(\begin{array}{c}
R^{R} \\
R^{I}
\end{array}\right)=\left(\begin{array}{c}
-\xi^{R} \\
\xi^{I}
\end{array}\right)-\left(\begin{array}{cc}
\left(\mathbf{A}^{[2]}-\hbar \omega \mathbf{1}\right) & \hbar \gamma \mathbf{1} \\
\hbar \gamma \mathbf{1} & -\left(\mathbf{A}^{[2]}-\hbar \omega \mathbf{1}\right)
\end{array}\right)\left(\begin{array}{c}
t_{\mathrm{opt}}^{R} \\
t_{\mathrm{opt}}^{I}
\end{array}\right) .
$$

The norm of this residual is then checked for convergence. Note that the full righthand-side and Jacobian is used for this calculation, and we thus have an a priori control of the convergence by specifying convergence threshold for this residual. 
If convergence is not met, the residuals are used to form new trial vectors, by use of the preconditioner

$$
\left(\begin{array}{c}
b^{R} \\
b^{I}
\end{array}\right)=\left(\begin{array}{cc}
\left(\mathbf{A}_{0}^{[2]}-\hbar \omega \mathbf{1}\right) & \hbar \gamma \mathbf{1} \\
\hbar \gamma \mathbf{1} & -\left(\mathbf{A}_{0}^{[2]}-\hbar \omega \mathbf{1}\right)
\end{array}\right)^{-1}\left(\begin{array}{c}
R^{R} \\
R^{I}
\end{array}\right),
$$

where $\mathbf{A}_{0}^{[2]}$ is a diagonal representation of the full Jacobian, retaining the difference in orbital energies in the zeroth-order approximation. While such preconditioning is not strictly necessary, it leads to faster convergence as the trial vectors are transformed to a form that points more closely to the directions in the abstract vector space necessary for the calculation of converged solution vectors.

Note that the above discussion have been focused on one single frequency, but there is no restriction to the number of frequencies that can be treated simultaneously. As the solution vectors of frequencies close to each other are very similar, especially far from resonance, it is in fact advantageous to calculate many at the same time, as the subspace required will be very similar. This approach also fits well with a parallel paradigm of computing, as many actions can be carried out on separate threads. Further, as the damping factor $\gamma$ is associated with the external frequency $\omega$ without reference to the explicit excited states, different lifetimes of the excited states can only be addressed if the density of state is sufficiently low, as we otherwise have a mixing of several transitions at an intermediate frequency.

\subsubsection{Comparison of the Lanczos and reduced subspace ap- proaches}

We will now compare two methods by which we can calculate complex polarizability with the CPP-CC approach: by an asymmetric Lanczos-chain-driven algorithm and a direct CPP solver in a reduced subspace. These approaches have different advantages and disadvatages, as will be discussed briefly:

- The Lanczos-chain-driven algorithm has the advantage of calculating the explicit excitation vectors, enabling a more in-depth analysis of the nature of the excited states and the possibility of broadening the different spectral features by different factors, if needed. The excitation vectors obtained at a CCSD level of theory can also be used for the calculation of triple corrections using CCSDR(3). Further, using the transition energies and moments, it is also possible to obtain total photo-ionization cross-section profiles by use of Stieltjes imaging [40]. However, the approach lacks an a priori control of the convergence and states of low intensities may be missed if care is not taken. In principle, it also has the disadvantage that all excited states should be calculated for the molecular response for any specific frequency, as can be seen by the sum in Eq. 3.20. Nonetheless, as only intense transitions close to the frequency under consideration gives a significant contribution, this is seldom a concern.

- The CPP-CC solver solver in an iteratively constructed subspace has a clear convergence with no ambiguity, and we can address any frequency region 
we desire. We thus have no need of calculating any features outside the region of interest, an advantage that is especially useful for the calculation of core excitations, as they are hidden so far into the excitation space. As no excited states are explicitly calculated, the density-of-states will only affect the number of iterations needed for convergence and thus offers little difficulty. As for the negative properties of this approach, it offers no explicit excited states if these are desired, and the possibility of studying the nature of the excitation is thus limited to studying the nature of the response vector at resonance (if the density-of-states is sufficiently small, and/or the damping factor is sufficiently small, the results should be very similar). It also means that we can not do perturbative corrections to energetics, i.e. no $\operatorname{CCSDR}(3)$ corrections are possible, and if a different (global) broadening parameter is desired, the properties needs to be re-calculated.

It is to be noted that all theoretical results presented in the following two sections have been obtained using a Lanczos-chain-driven approach, as this was the first computational scheme to be developed. 


\section{CHAPTER 4}

\section{Results and discussion}

In this chapter the performance of the developed damped coupled cluster linear response function utilizing a Lanczos-chain algorithm will be illustrated. As the scheme is of particular interest for calculating X-ray absorption spectra, owing to the important relaxation effects and the difficulty of treating the vast number of valence excitations in standard CC response theory, this will be the focus of this chapter. However, there are other applications for which the approach can be advantageous, and this chapter will end with a brief discussion of these situations.

\subsection{X-ray absorption spectroscopy}

We will first consider the theoretical aspects of core excitation processes, with focus on the two different roles of relaxation. In line with the discussion in Section 2.1.3 and the illustrative calculations in Section 2.2.3, the approach towards the correct physical description of the excitations is then evaluated in terms of finite basis sets effects, scalar relativistic effects, and the effects of truncation of the CC excitation manifold. The first aspect is illustrated for the NEXAFS spectrum of neon, and the latter two aspects for the NEXAFS spectrum of ethene, with comparisons made to previously published results [I-III]. The most reliable theoretical spectra obtained here and in previous studies are compared to experiment, and the remaining discrepancies are discussed. Finally, the performance of damped linear response CC and KS-DFT are compared, in order to assess the latter. This assessment is of importance in order to understand under which circumstances KS-DFT can be used, as the huge saving in computational costs compared to CC makes it a desirable option, but the lack of a reliable description of e.g. relaxation effects warrant caution.

Note that all CPP calculation have a common lifetime broadening of $\gamma=$ 
$1000 \mathrm{~cm}^{-1}$, yielding spectral features of a resolution expected to supersede experiment.

\subsubsection{Performance of the coupled cluster hierarchy}

For X-ray absorption processes, relaxation affects the molecule in two distinct physical manners, as a 'direct' relaxation and as a polarization/screening effect. The 'direct' relaxation originate from the reduced screening of the core as experienced by the valence electrons, and it is thus an attractive effect. The polarization/screening is instead a repulsive effect, resulting from the interaction between the excited electron and the valence electrons. Since the former effects dominates over the latter in terms of energetics, schemes by which only the 'direct' effect is accounted for. While this gives reasonably good spectra, the features becomes compressed and it is thus a method we will not pursue further.

Let us returning to the exact state linear response function of Eq. 3.20, from which the calculation of complex polarizability is in principle exact. This means that for any approximate state damped linear response functions, the relaxation effects depends only on the quality of the electronic structure method and is thus treated on par with electron correlation. In $\mathrm{CC}$, the electron correlation is included by excitations to virtual orbitals, and relaxation effects are thus included by the use of an appropriately large excitation manifold. Noting that an excitation manifold of single excitations can describe only the excitation of the core electrons, i.e. no relaxation effects, it is understood that at least an approximate doubles excitation manifold is necessary.

Before moving to this $N$-particle description, the basis set requirements of core excitations need to be determined. Since we are interested in correlated CC calculations, it can be expected that a basis set of triple- $\zeta$ quality is necessary. The basis set needs to be augmented also with diffuse functions, describing the excited state, and polarizing functions for the core, describing the relaxation of the core.

In order to determine the requirements, consider the NEXAFS spectrum of neon in Fig. 4.1, as compared against the experimental spectrum from Ref. [41]. The CPP-CCSD results were obtained using a basis set augmented by diffuse (aug-) functions specific for neon, and an additional set of generic Rydberg (6s6p) functions, giving a reasonable description of the excited state. Using this, the $\zeta$ level was varied as $\mathrm{X}=\mathrm{D}, \mathrm{T}, \mathrm{Q}$. In Fig. 4.1 the latter two calculations were obtained using additional core-polarizing functions, further improving the description of the core and relaxation effects. As is clear from the figure, the $\zeta$-level mainly influence the absolute energy, with smaller corrections to the spectral features. This is in line with results obtained for ethene in Paper II, where we concluded that a double- $\zeta$ basis set can give reasonable spectral features. However, in this study the excitation energies were consistently decreased for all improvements of the basis set, while for neon we observe a greater discrepancy to experimental values for the quadruple- $\zeta$ than for the triple- $\zeta$ basis sets. This trend was verified with additional calculations using a triple- $\zeta$ basis set with no core-polarizing functions, yielding a discrepancy of $0.79 \mathrm{eV}$, and using a quintuple- $\zeta$ basis set, for which the discrepancy 


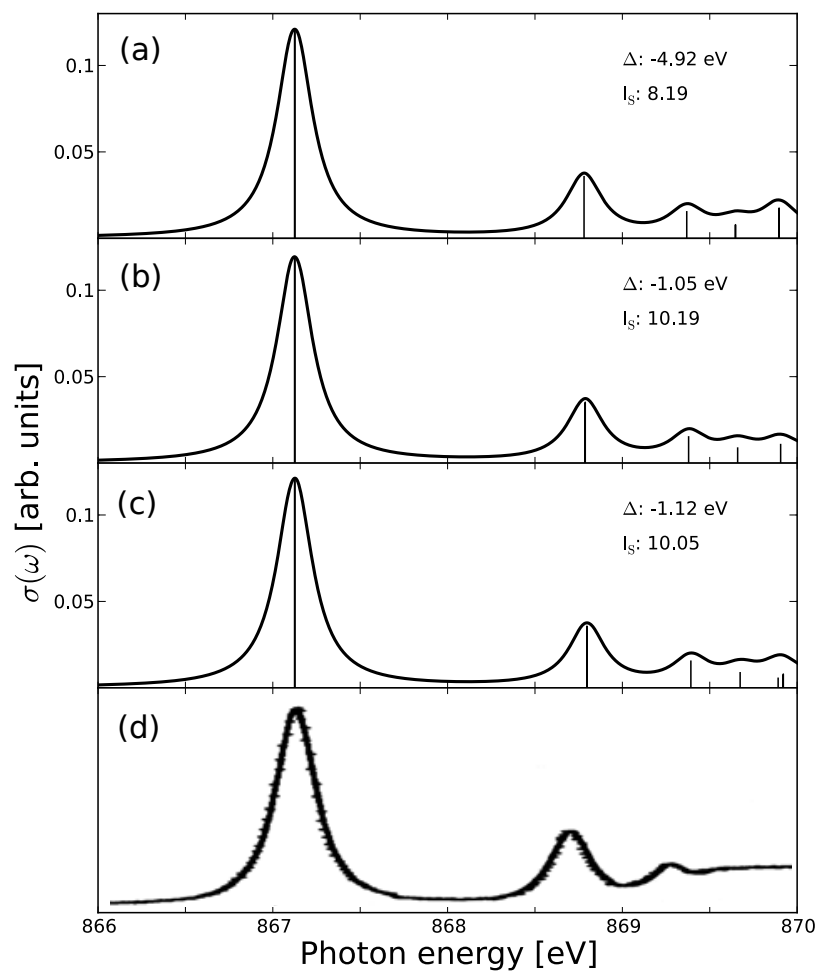

Figure 4.1. X-ray absorption spectra of neon as obtained with CPP-CCSD (a-c) and compared against experiment (d) [41]. Results are aligned against the first experimental peak by the shifts $\Delta$, and the total integrated absorption cross-section $\mathrm{I}_{\mathrm{S}}$ are also reported. The theoretical spectra were obtained using the basis sets (a) aug-cc-pVDZ, (b) aug-cc-pCVTZ, and (c) aug-cc-pCVQZ, all augmented with (6s6p) Rydberg functions. Lanczos chain length $j=5000$.

again is $0.83 \mathrm{eV}$. This non-monotonous convergence towards experimental values reflects the balance in description of the different region of the molecule, which is thus seen to vary. However, the absolute energetics is of less importance than the overall features.

In addition to the spectral features and absolute energies, we also report the total integrated absorption cross-section $\mathrm{I}_{\mathrm{S}}$. As was discussed in Section 3.2.2, this should, for a variational electronic structure method, be equal to the number of electrons in the system, and we see that the value moves towards 10 fo reach improvement in the basis set. From the calculation using a quintuple- $\zeta$ basis set we report an $\mathrm{I}_{\mathrm{S}}$ of 10.02. Finally, as the relaxation in CCSD is expected to be described by the multi-electron character of the excitation, we have calculated the double excitation $\left(T_{2}\right)$ character of the $1 s \rightarrow 3 p$ transition. These values are consistently observed in the range $8.0-8.1 \%$, indicating that the NEXAFS spectrum of neon has a rather small double excitation contribution. 
Moving on to the $N$-particle description, the carbon $K$-edge NEXAFS spectrum of ethene have been calculated using the CPP-CC hierachy CCS, CC2, CCSD, and CCSDR(3). The results are reported in Fig. 4.2, as compared against experiment [42]. Since the CCSDR(3) method only corrects the CCSD energetics and leaves the transition energies unaffected, the results are included as the CCSDR(3) shifts in the panel for CCSD. Finally, in order to study the nonvariational nature of $\mathrm{CC}$, the integrated absorption cross-sections $\mathrm{I}_{\mathrm{S}}$ are also reported. Similar studies of the CC hierarchy of NEXAFS spectra are found in Papers I and III, and we will now discuss each CC method in turn.

At the CCS level of theory, the error in absolute energy amounts to 10.09 $\mathrm{eV}$, consistent with previous findings of 7-21 eV. Comparing all these studies, we also observe a consistent behaviour in which the relaxation effects increase with the excitation energy, amounting to $7-11,14,16-17$, and $21 \mathrm{eV}$ for excitations from the core of carbon, nitrogen, oxygen and neon, respectively. In relation to the experimental excitation energies, this amounts to discrepancies of $2-4 \%$. More problematic, we observe that the spectral features are in bad agreement to experiment for all studies, and thus concludes that CCS is insufficient for core excitations. This was expected, as CCS lacks any relaxation effects.

Including approximate double excitations with the CC2 method, the error in absolute energy is decreased by over $7 \mathrm{eV}$ and large scale spectral features are improved, but the fine structure details are still unreliable. This is consistent with the findings in Papers I and II, in which CC2 can in some cases be observed to yield better absolute energies than CCSD (an effect of error cancellation between an incomplete treatment of double and no treatment of triple excitations), but the fine structure spectral features are shown to be inconsistent with experiment. However, for studies of chemical shifts Paper III demonstrated that the relative energy and intensity of excitations from chemically inequivalent carbon atoms was we reproduced at a $\mathrm{CC} 2$ level, yielding errors in energetics smaller than those of CCSD. It is thus concluded that CC2 can be a viable option for the study of intense features, but care must be taken for any fine structure details.

With the full double excitation manifold included in the CCSD method, the error in absolute energy is decreased further and, more importantly, the spectral features are now in excellent agreement with experiment. The CCSD energetics are then further improved by the CCSDR(3) method, approximately accounting for the triple excitations and decreasing the $1 s \rightarrow \pi^{*}$ transition energy by $0.71 \mathrm{eV}$. We note that the $\operatorname{CCSDR}(3)$ method requires well converged eigenvectors, thus requiring the Lanczos chain length to be large to ensure convergence of the pseudovectors. As a result of this, it is not always possible to obtain triple corrections by these means, and in Paper III we instead do this for two molecular systems, and estimate the correction for the remaining specimen to be approximately the same.

As illustrated in Fig. 2.1 there is an as of yet not treated dimension of quantum chemistry, namely relativistic effects. For $K$-edge spectroscopy these effects are scalar in nature, and they are thus accounted for by means of the second-order Douglas-Kroll-Hess Hamiltonian. Using this, we report positive shift in excitation energies of approximately $0.1 \mathrm{eV}$, consistent with findings in Papers I and III. 
The total integrated absorption cross-section is observed to undershoot the correct value for CCS, overestimate the necessary positive correction at a $\mathrm{CC} 2$ level and instead overshooting the correct value, and bringing it down slightly at a CCSD level. This again illustrates the non-variational nature of CC, and the remaining discrepancy can be understood as a combination of an insufficient treatment of the CC excitation manifold and an incomplete basis. Seeing the quite significant improvement at a quadruple- $\zeta$ level in Fig. 4.1, the latter effect can be expected to be more important.

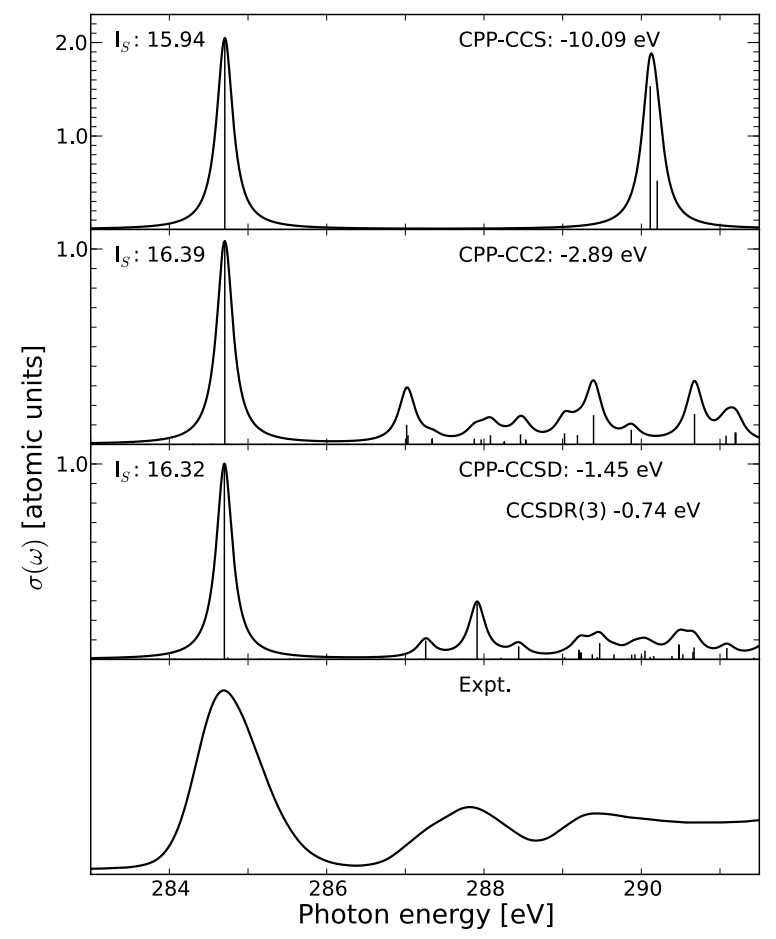

Figure 4.2. X-ray absorption spectra of ethene as obtained with a hierarchy of CPP$\mathrm{CC}$ methods and compared against experiment [42]. The results are aligned against the first experimental transition, with corresponding shifts given next to each model label. Included is also the total integrated absorption cross-section $\mathrm{I}_{\mathrm{S}}$. Basis set chosen as aug- cc-pCVTZ/cc-pVTZ for $\mathrm{C} / \mathrm{H}$, augmented by Rydberg (3d3p3d) functions at the centre of the $\mathrm{C}-\mathrm{C}$ bond. Lanczos chain lengths $j=6000$ and 8000 for $\mathrm{CC} 2$ and CCSD, respectively, and the full excitation space was utilized for CCS.

With these illustrative examples considered, we are now ready to evaluate the CPP-CC method in terms of comparing the most accurate theoretical excitation energies found here and in Papers I-III to experimental measurements, as determined for the most intense transitions. These values are reported in Table 4.1, where we also report the best non-relativistic CCSD energies, $T_{2}$ amplitudes and $\mathrm{I}_{\mathrm{S}}$ values. Computational details are found in the respective references. 


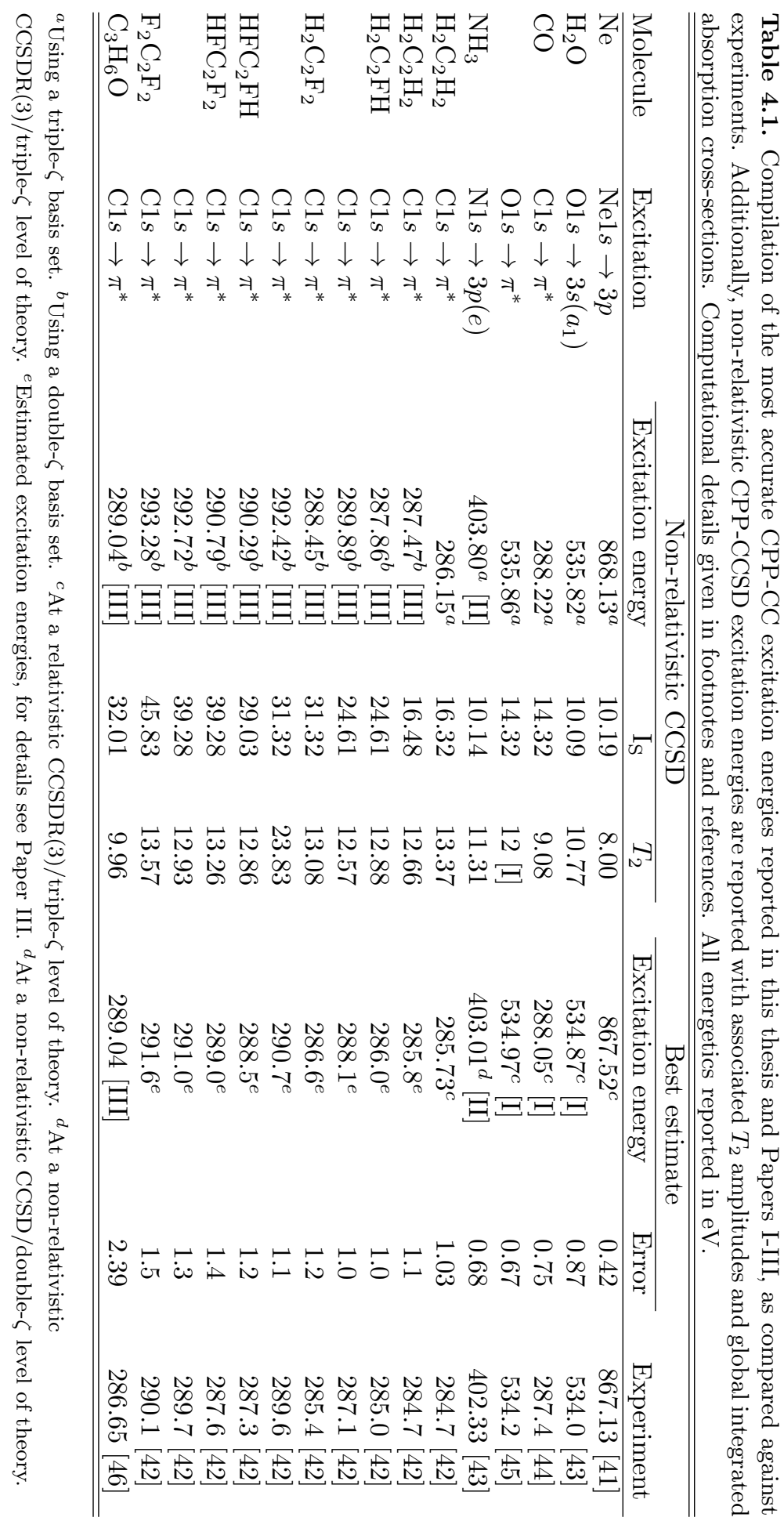


The remaining discrepancy in energetics are observed to be positive for all molecules, ranging from 0.42 to $1.5 \mathrm{eV}$ for a triple- $\zeta$ basis set, and amounting to 2.39 for acetone, for which only a double- $\zeta$ basis set was used. The latter value should be corrected for the basis set, triple excitations in the $\mathrm{CC}$ manifold and scalar relativistic effects, as was done for fluoroethenes in the same Paper III. Resulting accuracy is expected to be similar to that of the fluoroethenes. The origin of the remaining discrepancy can be understood as a combination of an incomplete basis set and coupled cluster excitation manifold, and we note that it appears to be the largest for the fluoroethenes, increasing with the size of the systems. However, these theoretical values were estimated from ethene and 1,1difluoroethene, as the larger molecules were to computationally demanding to be viable. It is thus likely that the estimated correction was unable to properly account for the substantial influences of the very electronegative fluorine atoms, which in turn are the origin of the large chemical shifts. Nonetheless, properly relativistic $\operatorname{CCSDR}(3)$ calculation on ethene utilizing a triple- $\zeta$ basis set resulted in a discrepancy of $1.03 \mathrm{eV}$, so the estimates should still be reasonable.

Moving on to the non-relativistic CCSD calculations, Fig. 4.3 shows scatter plots of error in absolute energy versus either the number of the electrons or the $T_{2}$ ampiltudes. For the number of electrons we also include the estimation made by the total integrated absorption cross-section $\mathrm{I}_{\mathrm{S}}$, and we note that the $\mathrm{I}_{\mathrm{S}}$ values are more accurate for the calculations utilizing a triple- $\zeta$ basis set, in line with the results presented in Fig. 4.1. There is a clear grouping of the double- $\zeta$ and triple- $\zeta$ results in terms of error in energetics, as is expected. Further, the double- $\zeta$ results has an ordering that would indicate that the error increases with the number of electrons. This is not unreasonable, as the number of electrons that needs to be relaxed increases, as does the total correlation energy, and this may increase the computational difficulty in these terms. However, this interpretation should not be taken too literal, especially as the triple- $\zeta$ results lacks this ordering.

For the $T_{2}$ amplitudes, we note one clear grouping of the double- $\zeta$ results, corresponding to carbon $1 s \rightarrow \pi^{*}$ transitions in fluoroethenes. There is also a slight increase of the $T_{2}$ amplitudes along with the error in energetics, which in turn correlates somewhat with the number of electrons, which could fit the previous discussion of increasing relaxation. But again, too much should not be read into this and we note that the slope of this line is indeed small. We also note one clear outlier at $24 \%$, corresponding to excitation of a core electron at the carbon bond to two fluorine in 1,1-difluroethene. This indicates strong relaxation effects for this excitation, possible a result of the valence electrons experiencing strong attraction to the very electronegative fluorine and the less screened carbon core, simultaneously. Further, we note a grouping of the triple- $\zeta$ results that are consistent with a correlation between the error and $T_{2}$ amplitudes, but the scattering is large and the number of data points small. 

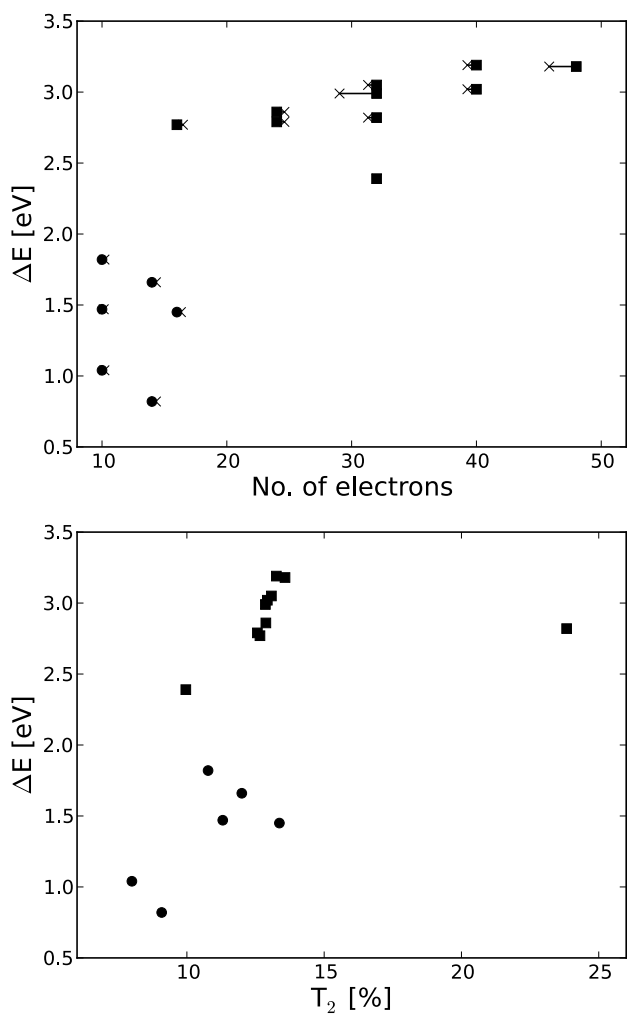

Figure 4.3. Scatter plot of the CCSD results in Table 4.1, utilizing a triple- $\zeta$ (circles), and a double- $\zeta$ (squares) basis set. For details on the determination, see Table 4.1 and references therein. Upper panel: error in energetics versus the number of electrons or the $\mathrm{I}_{\mathrm{S}}$ values (crosses). Lower panel: error in energetics versus $T_{2}$ amplitudes.

\subsubsection{Treating larger molecules}

While CC is a highly accurate electronic structure method it is also very expensive, as was seen in the scaling of Table 2.1. As such, CC calculation are viable only for small systems, and we would thus need alternative methods using which XAS spectra can be calculated in a reliable manner.

In general, Kohn-Sham density functional theory (KS-DFT) offers an alternative with a very low computational cost, even if it is less predictive than CC. We thus seek to compare the performance of DFT methods to that of CC, to see if the former is a viable option. For this, we will turn to a CPP formulation of time-dependent Kohn-Sham DFT, similar to the approach described Section 3.2.3, and as can be found in e.g. Ref. [39]. This is not the only formulation of timedependent DFT used for XAS, and a discussion of a more standard formulation is found in Ref. [2]. 


\section{Time-dependent Kohn-Sham density functional theory}

What follows is a brief description of KS-DFT and the application of a timedependent counterpart for the calculation of XAS spectra, for further details, see e.g. Refs. $[2,8,9]$.

For ground state properties, KS-DFT offers an appealing alternative to wave function-based electronic structure methods, owing to the large reduction in computational cost. The method is based on the electron density rather than the electron wave functions, and thus has a lower degree of freedom which reduce the costs. However, the method suffers from one potentially sever disadvantage, namely the lack of an exact so-called exchange-correlation functional. If this was known KS-DFT would be exact, but what is the case is instead that functionals are tailored for different properties, affecting the predictability of the method. Nonetheless, it offers a method that is computationally advantageous, and has been one of the greatest boon to the scientific field of quantum chemistry.

For time-dependent properties, we move to a time-dependent formulation of KS-DFT. In this thesis a CCP formulation of this methodology, CPP-DFT, is utilized, as can be found in Ref [39]. In the methodology an adiabatic approximation is commonly assumed, replacing the time-dependent functional with the static case. This is an approximation that should be reasonable as long as the variations of the correlation-exchange in time is small. Additionally, the theoretical foundation of the method have been criticized, with claims that the method is not predictive [47-49]. Self-interaction errors also yields large error in core excitation energetics, but these effects are well understood and schemes have been developed to account for them both for binding energies [50] and XAS spectra [51]. Of greater concern is that CPP-DFT is in principle a single-determinant method, and could thus be expected to experience the same problem of relaxation as CPP-CCS.

With all of these concerns in mind, time-dependent KS-DFT still offers a computationally advantageous method, and studies on XAS spectra yields excellent results nonetheless [2]. The method is especially interested for organic molecules with saturated carbon chains, in which the local nature of the excitations enables the spectrum to be calculated by the summation of the spectra of parts of the molecule - as by the building-block principle, enabling the treatment of even larger systems. As presented in Ref. [52], CPP-DFT utilized for XAS calculations needs a Coulomb attenuated functional, as the excitation is of a character similar to charge transfer. In this study a CAM-B3LYP functional was tailored for XAS calculation, and we will thus use the parameters determined in therein: $\alpha=0.19$, $\beta=0.81$, and $\gamma=0.33$.

\section{Relaxation in time-dependent coupled cluster and Kohn-Sham density funtional theory}

In Paper III the capacity of CPP-CC and CPP-DFT for reproducing the chemical shifts of $1 s \rightarrow \pi^{*}$ transitions were evaluated for a number of fluorine-substituted ethenes was evaluate. In addition to these methods, four-component static-exchange calculations were also performed [53], but this will not be treated any further.

For this evaluation vinylflourine $\left(\mathrm{HFC}_{2} \mathrm{H}_{2}\right)$, 1,1-difluoroethene $\left(\mathrm{F}_{2} \mathrm{C}_{2} \mathrm{H}_{2}\right)$ and 
trifluoroethene $\left(\mathrm{F}_{2} \mathrm{C}_{2} \mathrm{FH}\right)$ were chosen as practical examples, with experimental carbon $K$-edge NEXAFS spectra found in Ref. [42]. For these system, the chemical shifts are substantial owing to the influence of the very electronegative fluorine atoms - the valence electrons are strongly attracted and thus reduced the screening experienced by the carbon core electrons, increasing the binding energies. As such, the relaxation effects following core excitations are very site-specific and this thus offers a good test of theoretical methods. The molecules are also good examples of the important class of $\pi$-conjugated systems, interesting especially for the study of bio-molecules. These molecules possess easily identified $1 s \rightarrow \pi^{*}$ transitions, which was the focus of the comparative study.

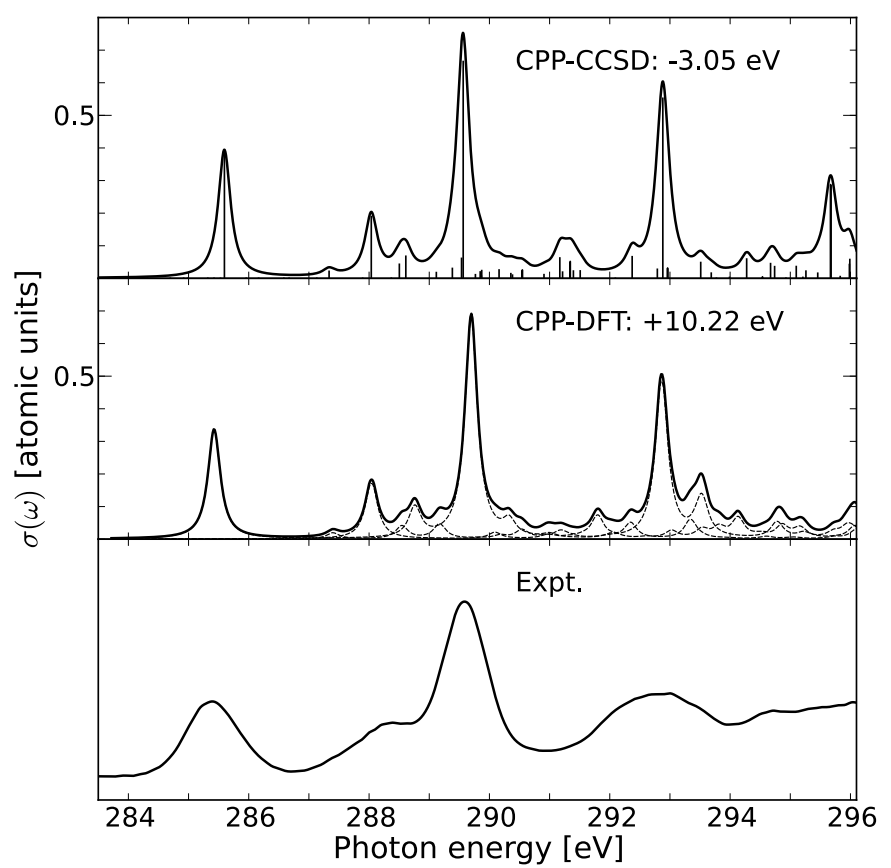

Figure 4.4. Carbon $K$-edge spectra of 1,1-difluoroethene obtained by CPP-DFT and Lanczos CPP-CCSD calculations and compared to experiment.Theoretical $1 s \rightarrow \pi^{*}$ have been align to experiment [42]. Basis set aug-cc-pCVDZ for carbon, cc-pVDZ for the remaining elements, and additional Rydberg $(3 s 3 p 3 d)$ have been centred at the center of the $\mathrm{C}-\mathrm{C}$ bond. Lanczos chain length $j=4000$.

The carbon $K$-edge NEXAFS spectrum of 1,1-difluoroethene have been calculated at a CPP-CCSD and CPP-DFT level, and the results are found in Fig. 4.4, as compared to experiment. As can be seen, the spectral features of DFT match those of CCSD very well, and the chemical shift seems to be well reproduced for both methods. Comparisons for the spectra of vinylfluoride and trifluoroethene can be found in Paper III, showing the same behaviour for DFT and CCSD as for 1,1-difluoroethene. 
Focusing in the $1 s \rightarrow \pi^{*}$ transitions, the theoretical and experimental chemical shifts are reported in Table 4.2. Included are also chemical shifts obtained with ground-state HF and KS-DFT, the four-component STEX method, CPPCCS and CPP-CC2, as well as an estimation of CCSDR(3) shifts. Ground-state HF, STEX and CPP-CCS are all seen to result in large discrepancies when compared to experimental values and will not be considered further. Using CPP-CC2 or CPP-CCSD, we report excellent agreement with experiment, with a discrepancy of $0.03-0.09$ and $0.07-0.23 \mathrm{eV}$, respectively. The latter shift is further improved by the CCSDR(3) estimation, yielding errors in chemical shifts of $\leq 0.1$ $\mathrm{eV}$. The excellent agreement of $\mathrm{CC} 2$ is somewhat surprising,given the unreliable fine structure features of this method. This is obtained also for the three molecules studied here, and we thus see that CC2 is suitable for studies of intense chemical shifts, but not for fine structure features. The obtained values actually outmatch those of CCSD.

For CPP-DFT we note a large shift in energetics, resulting from self-interaction errors. This shift can be accounted for at will, see e.g. Ref. [51], but we have refrained for doing so in this study. The reason for this is that the self-interaction error is well understood, amounting to approximately $10 \mathrm{eV}$ for the carbon $K$ edge. It can thus be accounted for, and what is more important is that the spectral features, i.e. relative energies and intensities, are well reproduced. With a chemical shift of $0.03-0.08 \mathrm{eV}$. This is to be compared to the shift in Kohn-Sham orbital energies, yielding chemical shifts overestimating experiment by $0.24-0.47 \mathrm{eV}$. We thus manage to reproduce the chemical shifts for these systems with a greater accuracy than CCS. The reason for this is not well understood at the moment, as the method is of a single determinant nature and should thus be unable to reproduce relaxation effects. It is to be noted that the largest discrepancy, of 0.08 $\mathrm{eV}$, is obtained for 1,1-difluoroethene, the molecule exhibiting the largest chemical shift and for which the $T_{2}$ amplitudes are obtained as $13.1 \%$ and $23.8 \%$. It could be expected that systems which have relatively small $T_{2}$ amplitudes of approximately the same values are better reproduced, as the relaxation effects would be of the same magnitude for all excitations. But even with the mentioned difference of $13.1-23.8 \%$, the discrepancy amounts only to $0.08 \mathrm{eV}$. This surprising feature is currently under investigation.

\subsection{Other applications using damped coupled clus- ter response theory}

As explained in Chapter 3, molecular response theory offers a framework for the theoretical treatment of a plethora of molecular properties. From the complex polarizability it is possible to obtain the absorption and dispersion of ultraviolet, visible and $\mathrm{X}$-ray radiation, as well as calculating dipole-dipole dispersion coefficients. We will now discuss the interaction of ultraviolet and visible light with pyrimidine, as well as the calculation of the dipole-dipole dispersion coefficient for the benzene dimer. The material presented is taken from Paper II, where further details can be found. 
Table 4.2. Carbon chemical shifts $(\mathrm{eV})$ of the $1 s \rightarrow \pi^{*}$ transitions in vinylfluoride, 1,1-difluoroethene, and trifluoroethene. Orbital energy differences are denoted by $\Delta \mathrm{E}_{1 s}$ and DFT and CC results refer to the CPP approach.

\begin{tabular}{lccc}
\hline \hline Method & $\mathrm{H}_{2} \mathrm{C}_{2} \mathrm{FH}$ & $\mathrm{H}_{2} \mathrm{C}_{2} \mathrm{~F}_{2}$ & $\mathrm{HFC}_{2} \mathrm{~F}_{2}$ \\
\hline$\Delta \mathrm{E}_{1 s, \mathrm{HF}}$ & 2.46 & 4.92 & 2.49 \\
$\Delta \mathrm{E}_{1 s, \mathrm{KS}}$ & 2.36 & 4.67 & 2.34 \\
$\mathrm{STEX}$ & 1.97 & 3.76 & 1.78 \\
$\mathrm{DFT}$ & 2.16 & 4.28 & 2.13 \\
$\mathrm{CCS}$ & 1.27 & 2.47 & 1.19 \\
$\mathrm{CC} 2$ & 2.17 & 4.17 & 2.01 \\
$\mathrm{CCSD}$ & 2.03 & 3.97 & 1.93 \\
Est. CCSDR $(3)$ & 2.1 & 4.1 & 2.0 \\
\hline Expt [42] & 2.1 & 4.2 & 2.1 \\
\hline \hline
\end{tabular}

Reprinted with permission from [III]. (C)2013 AIP Publishing LLC.

\subsubsection{Absorption and dispersion of ultraviolet/visible radi- ation}

As established in Section 3.1.2, the real and imaginary part of the complex polarizability corresponds to dispersion and absorption of incoming light, enabling a simultaneous treatment of the frequency-dependent refraction and attenuation of the radiation. The behaviour of these polarizabilities are illustrated for a single peak in Fig. 3.1, and for a real calculation of pyrimidine, $\mathrm{C}_{4} \mathrm{~N}_{2} \mathrm{H}_{4}$, in Fig. 4.5.

The absorption spectrum is obtained from the imaginary polarizability as by Eq. 3.22, analogous to the case of X-ray radiation. In Paper II the performance of the CPP-CC approach was compared to standard linear response CC, yielding identical results with the exception of a single missing feature in the standard approach. This is, however, an artifact of the implementation, and by no means signify a flaw in the theory itself. In terms of computational costs, the Lanczos algorithm required slightly less iterations, making it marginally faster, but this situation would change if the density of states increases. This is especially important for relativistic calculations, owing to the large number of triplet states that needs to be converged in order to get to high-intensity singled states. The difference between the approaches follows as a bottom-up approach need to converge all roots, regardless of the intensity, while the Lanczos algorithm are able to get correct global features using less iterations. This difference is even more significant if a CPP solver is used, as no roots are required and an arbitrary frequency region is directly targeted.

From the real part of the polarizability the index of light refraction can be found according to

$$
n(\omega)=1+2 \pi N \bar{\alpha}^{R}(\omega),
$$

where $N$ is the molecular number density and local field effects and intermolecular interactions are disregarded. As can be seen in Fig. 4.5, the behaviour of the 
frequency-dependent refractive index is more complicated that that of Fig. 3.1, as the density of states is higher. Further, the index index at around $8.4 \mathrm{eV}$ is negative, corresponding to the situation where the phase velocity of the radiation is greater than the speed of light. This behaviour can be difficult to measure, however, due to the relatively strong absorption for this frequency region.

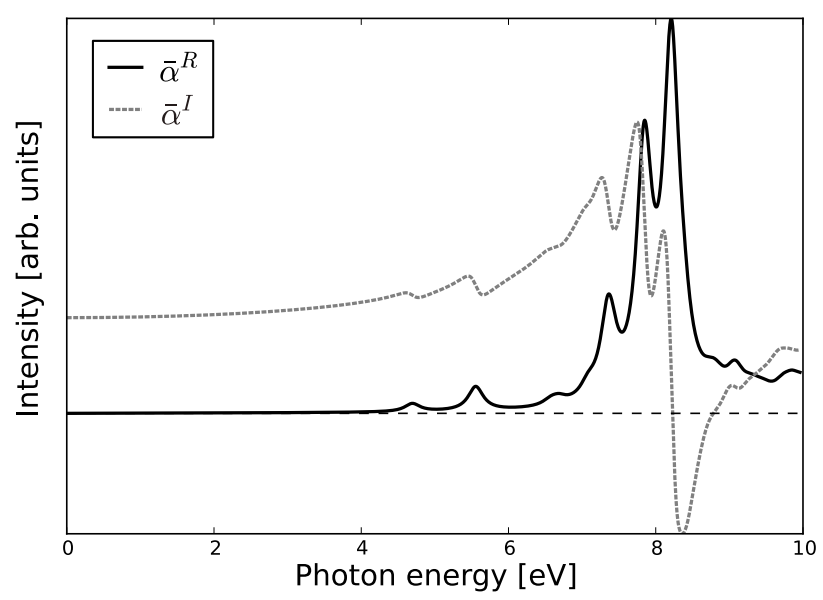

Figure 4.5. Real and imaginary part of the isotropic polarizability for pyrimidine, as obtained at the CCSD level of theory. The basis set was chosen as cc-pVDZ+ [54] with additional Rydberg $(3 s 3 p 3 d)$ augmentation at the centre of charge, and results were obtained using a Lanczos chain length $j=800$.

\subsubsection{Dipole-dipole dispersion coefficients}

By letting the optical frequency be identical to zero and instead varying the damping factor, it is possible to calculate the electric dipole polarizability of an imaginary frequency, $\bar{\alpha}(i \omega)$. The behaviour of this property is illustrated in Fig. 4.6, for benzene. From this property, the long-range dispersion interaction energy between two systems $A$ and $B$ can be calculated by the Casimir-Polder integral formula [55]

$$
C_{6}=\frac{3}{\pi} \int_{o}^{\infty} \bar{\alpha}^{A}(i \omega) \bar{\alpha}^{B}(i \omega) d \omega
$$

and the interaction energy in the van der Waals region is given as

$$
\Delta E=-\frac{C_{6}}{R^{6}}
$$

Using a Gauss-Legendre integration scheme, the $C_{6}$ coefficients were determined for methane and benzene dimers [II]. By use of the zero-point vibrational average correction the theoretical results were corrected for anharmonicity in the potential 
and polarizability, and the results were then compared to high-quality experimental estimates. Surprisingly, the discrepancy was found to be quite large, amounting to $3-8 \%$, and we have some concerns about the accuracy of the experimental estimates. However, this issue has not been pursued further, and it is possible that more elaborate calculations may resolve the issue.

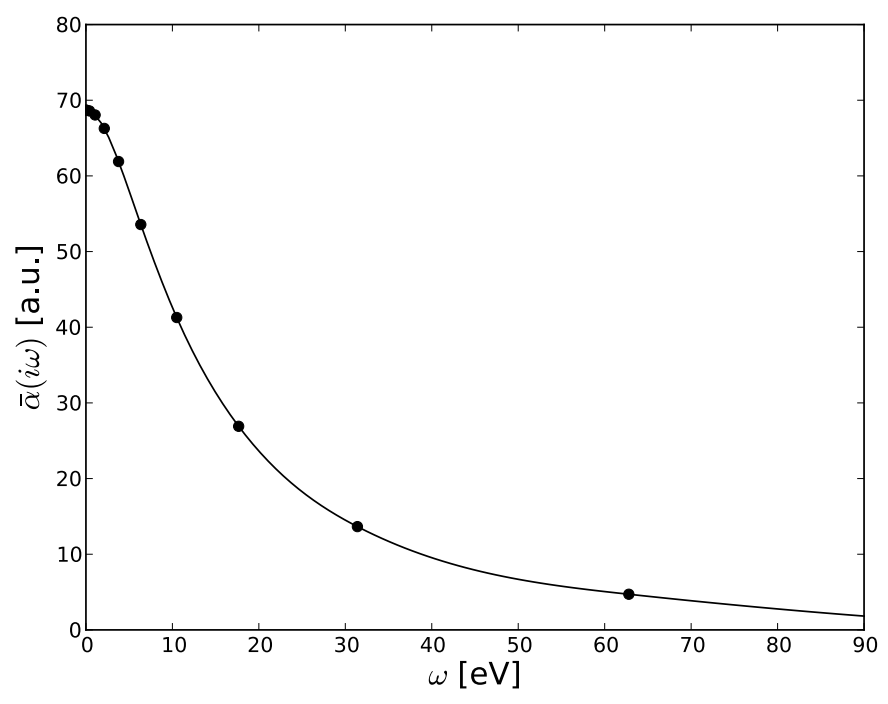

Figure 4.6. Isotropic average of the polarizability $\bar{\alpha}(i \omega)$ of benzene as a function of the imaginary frequency. Obtained at a CCSD/Sadlej-pVTZ level of theory. Reprinted with permission from [II]. (C)2012 American Chemical Society. 


\section{CHAPTER 5}

\section{Conclusions}

The damped linear response function, or complex polarization propagator (CPP) has been implemented in the coupled cluster (CC) approximation. In this framework, the calculation of complex polarizabilities by the hierarchy CCS, CC2 and CCSD are available, for which errors in the property calculations result only from the truncation of the coupled cluster manifold. In this CPP-CC approach, the calculation of molecular properties by two different methods has been discussed: an asymmetric Lanczos-chain driven algorithm and a direct CPP solver. The former approach enables the calculation of eigenvectors which can be used to characterize the excitations and, in the case of CCSD, correct the energetics by the CCSDR $(3)$ approach. With the direct CPP solver such corrections are unavailable, but the approach has an a priori control of the convergence and the ability to target arbitrary frequency regions without treating of any explicit excitations.

The performance of the CPP-CC method has been demonstrated for near-edge $\mathrm{X}$-ray absorption fine structure spectra of neon, water, carbon monoxide, ammonia, acetone and a number of fluorine-substituted ethenes. This spectroscopy is computationally challenging, owing to strong relaxation effects. It has been demonstrated that the relaxation effects amount to $7-21 \mathrm{eV}$ and require double excitations in the $\mathrm{CC}$ manifold in order to be accounted for. By approximate doubles included using the CC2 method, fine structure features are unreliable, but the intense features are still well reproduced. At a CCSD level of theory spectral features are in excellent agreement with experiment, and using a sufficiently flexible basis set and correcting for triple excitation and scalar relativistic effects, absolute energetics are reported with discrepancies of $0.4-1.5 \mathrm{eV}$ as compared to experiment. While these discrepancies are larger than for valence excitations, we note that the energy scale is of a different magnitude and the relative discrepancy is thus quite small. The remaining discrepancy is a mixture of the finite basis sets and truncation of the $\mathrm{CC}$ excitation manifold. 
The developed method has also been compared to the computationally more feasible method of CPP Kohn-Sham density functional theory, evaluating the performance of this method for the calculation of X-ray absorption spectra. With a suitable choice of KS-DFT functional, an accuracy of the same order as CPP-CCSD is observed (aside from a larger error in absolute energy, this being a result of the well-known self-interaction error). The site-specific relaxation is well accounted for using both CCSD and KS-DFT, and the latter result is surprising but promising. Further studies on the behaviour for time-dependent KS-DFT are necessary in order to understand the reason for the excellent spectral features.

Further, the developed CPP-CC method can be used for the calculation of other properties than those corresponding to XAS, and calculations on the refractive index and absorption cross-section of ultraviolet and visible light for pyrimidine shows excellent agreement to established computational schemes. CPP-CC can be expected to be suitable also for these properties if the density of states is high, making standard response methods less viable. Finally, calculations on the dipole-dipole dispersion coefficients of methane and benzene dimers illustrates yet another application of the developed method. 


\section{Bibliography}

[1] P. A. M. Dirac. Proc. R. Soc. Lond. A Math. Phys. Sci., 123(792), 1929.

[2] N. A. Besley and F. A. Asmuruf. Phys. Chem. Chem. Phys., 12(12024), 2010.

[3] J. Stöhr. NEXAFS Spectroscopy. Springer, Berlin, 1992.

[4] G. Hähner. Chem. Soc. Rev., 35(1244), 2006.

[5] T. Fransson. M.Sc. Thesis. Linköping, Sweden, LITH-IFM-A-EX-11/2527SE, 2011.

[6] T. Helgaker, P. Jørgensen, and J. Olsen. Molecular electronic-structure theory. John Wiley \& Sons Ltd., 2000.

[7] A. Szabo and N. S. Ostlund. Modern quantum chemistry: Introduction to advanced electronic structure theory. Dover Publications, 1996.

[8] R. Bast and P.-O. Widmark (editors). European Summerschool in Quantum Chemistry 2013 Book II. 2013.

[9] F. Jensen. Introduction to computational chemistry, 2nd Ed. John Wiley \& Sons Ltd., 2006.

[10] T. H. Dunning. J. Chem. Phys., 90(2), 1989.

[11] D. E. Woon and T. H. Dunning. J. Chem. Phys., 103(11), 1995.

[12] R. A. Kendall, T. H. Dunning, and R. J. Harrison. J. Chem. Phys., 96(9), 1992.

[13] K. Kaufmann, W. Baumeister, and M. Jungen. J. Phys. B-At. Mol. Opt., $22(2223), 1989$. 
[14] P. Strange. Relativistic quantum mechanics. Cambridge University Press, 1998.

[15] M. Douglas and B. A. Hess. Ann. Phys. (New York), 82(89), 1974.

[16] G. Jansen and B. A. Hess. Phys. Rev. A, 39(6016), 1989.

[17] P. Norman, B. Schimmelpfennig, K. Ruud, H. J. A. Jensen, and H. Ågren. J. Chem. Phys., 116(6914), 2002.

[18] P. Norman. Phys. Chem. Chem. Phys., 13(20519), 2011.

[19] G. D. Purvis. J. Chem. Phys., 76(4), 1982.

[20] K. L. Bak, J. Gauss, P. Jørgensen, J. Olsen, T. Helgaker, and J. F. Stanton. J. Chem. Phys., 114(15), 2001.

[21] T. H. Dunning. J. Phys. Chem. A, 104(40), 2000.

[22] O. Christiansen, H. Koch, and P Jørgensen. Chem. Phys. Lett., 243(5), 1995.

[23] O. Christiansen, H. Koch, and P. Jørgensen. J. Chem. Phys., 105(4), 1996.

[24] O. Christiansen, H. Koch, and P. Jørgensen. J. Chem. Phys., 103(17), 1995.

[25] A. B. Trofimov, G. Stelter, and J. Schirmer. J. Chem. Phys. , 117(14), 2002.

[26] S. Sauer, M. Schreiber, M. Silva-Junior, W. Thiel, and D. Mu. J. Chem. Theory Comp., 5(3), 2009.

[27] M. Silva-Junior, S. Sauer, M. Schreiber, and W. Thiel. Mol. Phys., 108(3), 2010.

[28] M. Schreiber, M. Silva-Junior, S. Sauer, and W. Thiel. J. Chem. Phys., 128(13), 2008.

[29] C. E. Moore. Atomic Energy Levels: As Derived From the Analyses of Optical Spectra. Natl. Bur. Stand. (U.S.) Circ. No. 467 (U.S. GPO, Washington, D.C., 1949), Vol. 1.

[30] T. Helgaker, S. Coriani, P. Jørgensen, K. Kristensen, J. Olsen, and K. Ruud. Chem. Rev., 112(543), 2012.

[31] P. Norman, K. Ruud, and T. Saue. Lecture notes from Quantum chemical winter school: Response properties of molecular materials. Chamonix, France, 2010 .

[32] B. H. Bransden and C. J. Joachain. Quantum mechanics, 2nd Ed. Pearson Education Limited, 2000.

[33] P. A. Tipler and R. Llewellyn. Modern physics, 4th Ed. W.H. Freeman \& Company, 2002. 
[34] O. Christiansen, P. Jørgensen, and C. Hättig. Int. J. Quantum Chem., 68(1), 1998.

[35] Z. Bai, J. Demmel, J. Dongarra, A. Ruhe, and H. van der Vorst. Templates for the solution of algebraic eigenvalue problems: A practical guide. Soc. Ind. Math., 1987.

[36] P. Seidler, M. B. Hansen, W. Gyorffy, D. Toffoli, and O. Christiansen. J. Chem. Phys., 132(16), 2010.

[37] J. Kauczor, P. Norman, O. Christiansen, and S. Coriani. Submitted to J. Chem. Phys.

[38] S. Villaume, T. Saue, and P. Norman. J. Chem. Phys, 133(064105), 2010.

[39] J. Kauczor, P. Jørgensen, and P. Norman. J. Chem. Theory Comput., 7(1610), 2011.

[40] J. Cukras, S. Coriani, P. Decleva, O. Christiansen, and P. Norman. J. Chem. Phys., 138(094103), 2013.

[41] M. Coreno, R. Avaldi, R. Camilloni, K. C. Prince, M. de Simone, J. Karvonen, R. Rolle, and S. Simonucci. Phys. Rev. A, 59(2494), 1999.

[42] R. McLaren, S. A. C. Clark, I. Ishii, and A. P. Hitchcock. Phys. Rev. A, 36(1683), 1987.

[43] J. Schirmer, A. B. Trofimov, K. J. Randall, J. Feldhaus, A. M. Bradshaw, Y. Ma, C. T. Chen, and F. Sette. Phys. Rev. A, 47(1136), 1993.

[44] Y. Ma, C. T. Chen, G. Meigs, K. Randall, and F. Sette. Phys. Rev. A, 44(1848), 1991.

[45] R. Püttner, I. Dominguez, T. J. Morgan, C. Cisneros, R. F. Fink, E. Rotenberg, T. Warwick, M. Domke, G. Kaindl, and A. S. Schlachter. Phys. Rev. A, 59(3415), 1999.

[46] K. C. Prince, R. Richter, M. de Simone, M. Alagia, and M. Coreno. J. Phys. Chem. A, 107(1955), 2003.

[47] J. Schirmer and A. Dreuw. Phys. Rev. A, 75(022513), 2007.

[48] N. T. Maitra, R. van Leeuwen, and K. Burke. Phys. Rev. A, 78(056501), 2008.

[49] J. Schirmer and A. Dreuw. Phys. Rev. A, 78(056502), 2008.

[50] G. Tu, V. Carravetta, O. Vahtras, and H. Ågren. J. Chem. Phys., 127(174110), 2007.

[51] G. Tu, Z. Rinkevicius, O. Vahtras, H. Ågren, U. Ekström, P. Norman, and V. Carravetta. Phys. Rev. A, 76(022506), 2007. 
[52] U. Ekström, P. Norman, V. Carravetta, and H. Ågren. Phys. Rev. Let., 97(143001), 2006.

[53] U. Ekström, P. Norman, and V. Carravetta. Phys. Rev. A, 73(022501), 2006.

[54] R. Kendall, T. H. Dunning, and R. Harrison. J. Chem. Phys., 96(6796), 1992.

[55] H. Casimir and D. Polder. Phys. Rev., 73(360), 1948. 


\section{List of included Publications}

[I] Coupled-cluster response theory for near-edge x-ray-absorption fine structure of atoms and molecules

S. Coriani, O. Christiansen, T. Fransson and P. Norman.

Phys. Rev. A 85, 022507 (2012).

[II] Asymmetric-Lanczos-chain-driven implementation of electronic resonance convergent coupled-cluster linear response theory

S. Coriani, T. Fransson, O. Christiansen and P. Norman.

J. Chem. Theory Comput. 8, 1616 (2012).

[III] Carbon X-ray absorption spectra of fluoroethenes and acetone: A study at the coupled cluster, density functional, and static-exchange levels of theory

T. Fransson, S. Coriani, O. Christiansen and P. Norman.

J. Chem. Phys. 138, 000000 (2013). 


\section{Included Papers}

The articles associated with this thesis have been removed for copyright reasons. For more details about these see:

http://urn.kb.se/resolve?urn=urn:nbn:se:liu:diva-102177 